\title{
Entropy, Lyapunov exponents and escape rates in open systems
}

\author{
Mark Demers \\ Fairfield University, mdemers@fairfield.edu \\ Paul Wright \\ Lai-Sang Young
}

Follow this and additional works at: https://digitalcommons.fairfield.edu/mathandcomputerscience-

facultypubs

Copyright 2011 Cambridge University Press

http://journals.cambridge.org/abstract_S0143385711000344

\section{Peer Reviewed}

\section{Repository Citation}

Demers, Mark; Wright, Paul; and Young, Lai-Sang, "Entropy, Lyapunov exponents and escape rates in open systems" (2011). Mathematics Faculty Publications. 35.

https://digitalcommons.fairfield.edu/mathandcomputerscience-facultypubs/35

\section{Published Citation}

MARK F. DEMERS, PAUL WRIGHT and LAISANG YOUNG (2012, Published online September 2011). Entropy, Lyapunov exponents and escape rates in open systems. Ergodic Theory and Dynamical Systems, 32, pp 1270- 1301 doi:10.1017/S0143385711000344

This Article is brought to you for free and open access by the Mathematics Department at DigitalCommons@Fairfield. It has been accepted for inclusion in Mathematics Faculty Publications by an authorized administrator of DigitalCommons@Fairfield. For more information, please contact digitalcommons@fairfield.edu. 


\title{
Entropy, Lyapunov exponents and escape rates in open systems
}

\author{
MARK F. DEMERS $\dagger$, PAUL WRIGHT $\ddagger$ and LAI-SANG YOUNG $\S$ \\ $\dagger$ Department of Mathematics and Computer Science, Fairfield University, Fairfield, USA \\ (e-mail: mdemers@fairfield.edu) \\ $\ddagger$ Department of Mathematics, University of Maryland, College Park, USA \\ (e-mail: paulrite@math.umd.edu) \\ $\S$ Courant Institute of Mathematical Sciences, New York University, New York, USA \\ (e-mail:lsy@cims.nyu.edu)
}

(Received 3 February 2011 and accepted in revised form 6 April 2011)

\begin{abstract}
We study the relation between escape rates and pressure in general dynamical systems with holes, where pressure is defined to be the difference between entropy and the sum of positive Lyapunov exponents. Central to the discussion is the formulation of a class of invariant measures supported on the survivor set over which we take the supremum to measure the pressure. Upper bounds for escape rates are proved for general diffeomorphisms of manifolds, possibly with singularities, for arbitrary holes and natural initial distributions, including Lebesgue and Sinai-Reulle-Bowen (SRB) measures. Lower bounds do not hold in such a generality, but for systems admitting Markov tower extensions with spectral gaps, we prove the equality of the escape rate with the absolute value of the pressure and the existence of an invariant measure realizing the escape rate, i.e. we prove a full variational principle. As an application of our results, we prove a variational principle for the billiard map associated with a planar Lorentz gas of finite horizon with holes.
\end{abstract}

\section{Introduction}

This paper is about leaky dynamical systems or dynamical systems with holes. A generic setup consists of a triple $(f, M ; H)$, where $M$ is the phase space of a map or flow denoted by $f$, and $H \subset M$ is an open set. We refer to $(f, M)$ as a closed system and $H$ as the hole through which mass is allowed to escape from the system. More precisely, we follow trajectories in $M$ until they enter $H$. Once a point enters $H$, it leaves the system forever, i.e. we stop considering it.

Holes can be large or small. Small holes are often used to model small (unintended) leaks in physical systems; proximity of normalized surviving distributions to the physical measure of the closed system is a form of stability. More generally, the study of $(f, M ; H)$ can be viewed as the study of dynamics on non-invariant domains. As an example of why such studies are relevant, consider the following. It is well known that attractors are 
important because they capture the large-time behavior of dynamical systems, but invariant sets that are not attracting can substantially impact the qualitative behavior of a system as well: let $\Lambda \subset M$ be such a set, and $U \subset M$ a neighborhood of $\Lambda$. Then we may regard $H=M \backslash \bar{U}$ as the hole. Slow escape rates from such holes are known to impact the speed of correlation decay of the closed system.

Escape dynamics have been studied by many authors. We refer the reader to the partreview article [DY] which contains many references, and will mention explicitly works that are closer to the present paper as we go along. Most previous works have focused on specific systems, such as Anosov diffeomorphisms, interval and billiard maps. In this paper, we seek a general understanding for as large a class of dynamical systems as we can. Specifically, we seek to relate escape rate to a dynamical invariant called pressure, which, roughly speaking, measures the discrepancy between metric entropy and the sum of positive Lyapunov exponents. We now proceed to a discussion of what this paper is about.

Setting and questions. We begin with the simpler setting of a compact Riemannian manifold $M$ without boundary and a diffeomorphism $f$ which is at least $C^{1+\epsilon}$ for some $\epsilon>0$. In order to include applications to systems such as billiards, which are very important examples of dynamical systems of physical origin, we also allow $M$ to be the union of a (possibly open) Riemannian manifold and a singularity set $\mathcal{S}$, and $f$ to be piecewise smooth. Precise conditions on $\mathcal{S}$ and the behavior of $f$ near it will be introduced in $\S 2$. A Riemannian measure on $M$ (or $M \backslash \mathcal{S}$ ) is denoted by $\mu$ throughout. Unless otherwise stated, the hole $H$ is an arbitrary open set in $M$.

Let $m$ be a reference measure on $M$. We think of $m$ as the initial distribution of mass in the phase space before any escape takes place, and take the view that initial distributions related to $\mu$ are of particular physical interest. Notice that $m$ need not be $f$-invariant. Indeed, one can interpret the situation as follows: the escape of mass can begin before or after the closed system $f: M \circlearrowleft$ reaches a steady state. In the first case, $m$ is usually not invariant, and we assume it has a density with respect to $\mu$. In the second case, we take $m$ to be an SRB measure, which may be singular with respect to $\mu$.

A basic quantity of interest is the escape rate, defined to be $-\rho(m)$, where

$$
\rho(m)=\lim _{n \rightarrow \infty} \frac{1}{n} \log m\left(M^{n}\right)
$$

when the limit exists. Here, $M^{n}=\bigcap_{i=0}^{n} f^{-i}(M \backslash H)$ is the set of points which has not escaped by time $n$. In general, the limit in (1) may not exist, and we write $\rho$ and $\bar{\rho}$ for the $\lim _{n \rightarrow \infty}$ and $\limsup _{n \rightarrow \infty}$ of the quantity on the right-hand side. Notice that although $\rho(m)$ depends on $m$, all initial distributions uniformly equivalent to $m$ have the same escape rate, i.e. if $\varphi$ is a function with $1 / c \leq \varphi \leq c$ for some $c>0$, then $\rho(\varphi m)=\rho(m)$, and the same is true for $\rho$ and $\bar{\rho}$.

For an $f$-invariant Borel probability measure $v$ on $M$, the pressure of $v$, denoted by $P_{v}$, is defined to be

$$
P_{\nu}=h_{\nu}(f)-\int \lambda^{+} d \nu
$$

where $h_{v}(f)$ is the metric entropy of $(f, v)$ and $\lambda^{+}$is the sum of the positive Lyapunov exponents counted with multiplicity. We will write $\mathcal{P}_{\mathcal{G}}=\sup _{v \in \mathcal{G}} P_{\nu}$, where $\mathcal{G}$ is a collection of invariant measures. 
Given an open system $(f, M ; H)$, we define the survivor set to be the $f$-invariant set $\Omega:=\bigcap_{n \in \mathbb{Z}} f^{n}(M \backslash H) \dagger$. Let $\mathcal{I}=\mathcal{I}(\Omega)$ denote the set of $f$-invariant Borel probability measures supported on $\Omega$, and let $\mathcal{E} \subset \mathcal{I}$ be the subset of $\mathcal{I}$ consisting of ergodic measures. Assuming $\rho(m)$ is well defined, we say $\rho(m)$ satisfies a variational principle if

$$
\rho(m)=\mathcal{P}_{\mathcal{G}} \quad \text { for a suitable class of measures } \mathcal{G} \subset \mathcal{I} .
$$

Of interest also is whether the supremum in $\mathcal{P}_{\mathcal{G}}$ is attained, i.e. if there is a measure $v \in \mathcal{G}$ for which $P_{v}=\mathcal{P}_{\mathcal{G}}$. Obviously, one can also ask if $\rho(m)=P_{v}$ for some $v$, without mentioning any variational principles.

The ideas in the last paragraph were suggested by a number of previously known results, some of which are recalled below, but let us first summarize the questions to be addressed.

This paper seeks to address, for as large a class of dynamical systems as possible, the following three questions for natural initial distributions $m$.

(Q1) (Escape rate). Is the escape rate $-\rho(m)$ well defined?

(Q2) (Formula for escape rate). Is $\rho(m)=h_{v}(f)-\int \lambda^{+} d v$ for some $v \in \mathcal{I}$ ? The same question can be posed for $\underline{\rho}(m)$ and $\bar{\rho}(m)$.

(Q3) (Variational principle). Doess $\rho(m)$ satisfy a variational principle?

Partial answers are given for very general dynamical systems, and complete answers for a more restricted class, which includes many known examples. A concrete application to the leaky periodic Lorentz gas is mentioned explicitly.

\section{Earlier works.}

Theorem 1. [B] Consider a $C^{1+\epsilon}$ Axiom A diffeomorphism $f: M \circlearrowleft$ of a compact Riemannian manifold $M$. Let $\Lambda \subset M$ be a basic set, and let $\mathcal{I}=\mathcal{I}(\Lambda)$. Then $\mathcal{P}_{\mathcal{I}} \leq 0$, and $\mathcal{P}_{\mathcal{I}}=0$, if and only if $\Lambda$ is an attractor.

This is the first result that systematically relates the escape of mass to pressure: in the case where $\Lambda$ is an Axiom A attractor, no mass can escape from a neighborhood of $\Lambda$, and $\mathcal{P}_{\mathcal{I}}=0$; for non-attracting basic sets such as horseshoes, mass escapes at exponential rates and $\mathcal{P}_{\mathcal{I}}<0$. The number $\mathcal{P}_{\mathcal{I}}$ has been shown to be equal to the topological pressure of $f$ with respect to the potential $-\log \left|\operatorname{det}\left(D f^{u}\right)\right|$ on $\Lambda$; see $[\mathbf{B}]$ or $[\mathbf{W}]$ for more details.

The next result gives conditions under which the numerical value of $\mathcal{P}_{\mathcal{I}}$ is explicitly related to the rate of escape.

THEOREM 2. [Y1, Theorem 4] $\ddagger$ Let $f: M \circlearrowleft$ be a $C^{1+\epsilon}$ diffeomorphism of a compact Riemannian manifold $M$, and let $H \subset M$ be an open set. We assume:

(i) $\Omega$ is compact with $d(\Omega, \partial H)>0$; and

(ii) $\left.f\right|_{\Omega}$ is uniformly hyperbolic.

Then $\rho(\mu)$ is well defined and equals $\mathcal{P}_{\mathcal{I}}$.

In both of the settings above, $\mathcal{P}_{\mathcal{I}}=\mathcal{P}_{\mathcal{E}}$, and $\mathcal{P}_{\mathcal{I}}=P_{v}$ for some $v \in \mathcal{I}$. (The latter follows from the continuity of $x \mapsto \log \left|\operatorname{det}\left(\left.D f\right|_{E^{u}}\right)\right|$ and upper semicontinuity

$\dagger$ If $f$ is not invertible, we take $n \leq 0$ in the definition of $\Omega$.

$\$$ This result follows from the large deviation results in Theorem 1 (not Theorem 2) of [Y1]. Take $\varphi \equiv 1$ on a closed set $K$ and $<1$ on $M \backslash K$, where $\Omega \subset \operatorname{int}(K) \subset K \subset M \backslash H$, and $\xi \approx-\log \left|\operatorname{det}\left(\left.D f\right|_{E^{u}}\right)\right|$ on $\Omega$. 
of $v \mapsto h_{v}(f)$; see [B].) Thus, for uniformly hyperbolic survivor sets $\Omega$ with $d(\Omega, H)>0$, (Q1)-(Q3) have all been answered in the affirmative.

Several works went beyond Theorem 2 to give positive answers to (Q1) and (Q2) in a number of situations, including Anosov diffeomorphisms with Markov or small holes (with no requirement on $\Omega \cap \partial H$ ) [CM1, CM2, CMT], uniformly expanding maps admitting Markov partitions [CMS], piecewise expanding maps, and Collet-Eckmann maps of the interval with singularities [BDM]. (Q3) was partially addressed in [BDM, CM1, CMS]: a variational principle was proved for an associated dynamical system, namely the symbolic dynamics of the original map (but not for the map itself).

\section{Statement of results}

Three sets of results are stated.

- Section 2.1 contains partial answers to (Q3): lower bounds for $\underline{\rho}(\mathrm{m})$ are proved for very general dynamical systems; no results on upper bounds are reported.

- Sections 2.1 and 2.3 provide complete answers to (Q1)-(Q3) for systems admitting Markov tower extensions with some additional conditions.

- $\quad$ These results are applied to the periodic Lorentz gas with small holes (Theorem F).

2.1. Lower bounds on $\rho(m)$ for general dynamical systems. Our results in this subsection will assert, in essence, that

for very general dynamical systems, $\rho(m) \geq \mathcal{P}_{\mathcal{G}}$ for reasonable choices of $\mathcal{G}$.

Since $\mathcal{P}_{\mathcal{G}}$ decreases with $\mathcal{G}$, this inequality is not meaningful for $\mathcal{G}$ too small. Thus, the selection of a suitable $\mathcal{G}$ is an important part of the consideration. We start with $\mathcal{E}$, the set of ergodic invariant measures supported on the survivor set $\Omega$. To obtain $\mathcal{G}$, restrictions will be placed on $\mathcal{E}$ on account of:

I. the hole $H$;

II. the initial distribution $m$; and

III. singularities of the map $f$, if present.

We discuss these three types of restrictions separately. The conditions we impose are admittedly motivated by our proofs, but the fact that they lead to a full variational principle for a large class of dynamical systems (see $\$ 2.3$ ) suggests that these choices of $\mathcal{G}$ are reasonable.

Remark. One should keep in mind that the escape rate is defined by $-\rho(m)$ when interpreting the inequality $\underline{\rho}(m) \geq \mathcal{P}_{\mathcal{G}}$. Thus, a lower bound for $\underline{\rho}(m)$ provides an upper bound of $\left|\mathcal{P}_{\mathcal{G}}\right|$ for the escape rate.

In paragraphs I and II below, $f: M \circlearrowleft$ is a $C^{1+\epsilon}$ diffeomorphism; systems with singularities are discussed in paragraph III. Throughout the paper, $B(x, r)$ denotes the ball of radius $r$ in $M$ centered at $x \in M$, and $N_{\varepsilon}(\cdot)$ denotes the $\varepsilon$-neighborhood of a set in $M$.

I. Restrictions on $\mathcal{G}$ due to the hole $H$. The following definition gives a sense of which $v \in \mathcal{E}$ we think impact the escape rate. Define

$$
\begin{aligned}
\mathcal{G}_{H}=\{v \in \mathcal{E} \mid \text { the following holds for } \nu \text {-a.e. } x \text { : given any } \gamma>0, \\
\qquad \\
\left.\exists r=r(x, \gamma)>0 \text { such that } B\left(f^{i} x, r e^{-\gamma i}\right) \subset M \backslash H \text { for all } i \geq 0\right\}
\end{aligned}
$$


(a.e. denotes almost every). Notice that if $\nu \in \mathcal{E}$ has the property that for some $C, \alpha>0$, $v\left(N_{\varepsilon}(\partial H)\right) \leq C \varepsilon^{\alpha}$ for all $\varepsilon>0$, then $v$ is in $\mathcal{G}_{H}$ (see $\S 4.2$, paragraph 4).

The definition of $\mathcal{G}_{H}$ can be relaxed in many ways; in particular, it is not necessary for the entire ball $B\left(f^{i} x, r e^{-\gamma i}\right)$ to be in $M \backslash H$. We mention one formulation, leaving the reader to contemplate others: given $x \in M$, let $W_{\varepsilon}^{s}(x)$ denote the local stable manifold of $x$ of radius $\varepsilon$. We call an open set $O$ a $W^{s}$-neighborhood of $x$ if $O \cap W_{\varepsilon}^{s}(x) \neq \emptyset$ for every $\varepsilon>0$. All of our results remain valid if:

(O) in the definition of $\mathcal{G}_{H}, B\left(f^{i} x, r e^{-\gamma i}\right)$ is replaced by $f^{i}(O) \cap B\left(f^{i} x, r e^{-\gamma i}\right)$, where $O$ is a $W^{s}$-neighborhood of $x$.

II. Restrictions on $\mathcal{G}$ due to the initial distribution $m$. Two types of initial distributions are considered.

(A) Initial distributions with densities, possibly localized. Let $m=\mu_{\varphi}=\varphi \mu$, where $\varphi \geq 0$ is in $L^{1}(\mu)$. For such an initial distribution, we consider

$$
\mathcal{G}_{\varphi}=\left\{v \in \mathcal{E}: \exists c_{\nu}>0 \text { and an open set } Z \text { such that } v(Z)>0 \text { and }\left.\varphi\right|_{Z} \geq c_{\nu}\right\} .
$$

THEOREM A. Let $(f, M ; H)$ be as above. Then:

(i) $\rho(\mu) \geq \mathcal{P}_{\mathcal{G}_{H}}$;

(ii) more generally, $\underline{\rho}\left(\mu_{\varphi}\right) \geq \mathcal{P}_{\mathcal{G}_{H} \cap \mathcal{G}_{\varphi}}$.

Remark. Clearly, $\mathcal{G}_{\varphi}=\mathcal{E}$ if $\varphi \geq c$ for some $c>0$; thus (ii) reduces to (i). Here, we permit $\varphi$ to vanish on parts of $M$ provided it is measurable with ess $\inf (\varphi)>0$ on an open set of $M$. We do not claim that the restrictions imposed on $\mathcal{G}_{\varphi}$ are necessary, but if the support of $\varphi$ is localized in the phase space, invariant measures supported elsewhere are clearly irrelevant since they cannot be 'seen' by the initial distribution $\mu_{\varphi}$.

(B) SRB measures as initial distributions. In (A), $m=\mu_{\varphi}$ is not necessarily an invariant measure. If, however, a steady state is reached before the leak begins, then it would be natural to take $m$ to be an SRB measure $\mu_{\mathrm{SRB}}$, as we now do. For simplicity, we assume $\mu_{\mathrm{SRB}}$ has no zero Lyapunov exponents.

The challenge here is to identify a class of invariant measures $\mathcal{G}_{\mathrm{SRB}}$ that can be 'seen' by the SRB measure $\mu_{\mathrm{SRB}}$, which is often singular. We call $\Pi \subset M$ a $\mu_{\mathrm{SRB}}$-hyperbolic product set if the following hold.

(W.1) $\Pi=\left(\bigcup \Gamma^{u}\right) \cap\left(\bigcup \Gamma^{s}\right)$, where $\Gamma^{u}=\{\omega\}$ and $\Gamma^{s}=\left\{\omega^{\prime}\right\}$ are two sets of relatively open local unstable and stable manifolds such that each $\omega \in \Gamma^{u}$ intersects every $\omega^{\prime} \in \Gamma^{s}$ in precisely one point. In addition, there exist constants $C>0, \lambda<1$ such that

$\operatorname{diam}\left(T^{-n} \omega\right) \leq C \lambda^{n}$ for all $\omega \in \Gamma^{u}$ and $\operatorname{diam}\left(T^{n} \omega^{\prime}\right) \leq C \lambda^{n}$ for all $\omega^{\prime} \in \Gamma^{s}$,

where $\operatorname{diam}(\cdot)$ denotes the diameter of the unstable or stable manifold.

(W.2) $\left.\mu_{\mathrm{SRB}}\right|_{\Pi}(A)>0$ for every relatively open $A \subset \Pi$.

(W.3) There exists a constant $c_{\Gamma}>0$ such that for $\mu_{\mathrm{SRB}}-$ a.e. $\omega \in \Gamma^{u}$, the conditional probability of $\mu_{\mathrm{SRB}}$ on $\omega$ has density $\psi_{\omega} \geq c_{\Gamma}$. 
We remark that (W.3) is a general property of SRB measures [LY]; we have listed it separately only for emphasis. Define

$$
\mathcal{G}_{\mathrm{SRB}}=\left\{v \in \mathcal{E} \mid v(\Pi)>0 \text { for a } \mu_{\mathrm{SRB}} \text {-hyperbolic product set } \Pi\right\} .
$$

THEOREM B. Under the conditions above, $\underline{\rho}\left(\mu_{\mathrm{SRB}}\right) \geq \mathcal{P}_{\mathcal{G}_{H} \cap \mathcal{G}_{\mathrm{SRB}}}$.

Remark 1. Observe that if $f$ has an Axiom A attractor $\Lambda$ and $\mu_{\mathrm{SRB}}$ is the SRB measure on the attractor, then $\mathcal{G}_{\mathrm{SRB}}$ imposes no restriction whatsoever on $v \in \mathcal{E}$, i.e. $\mathcal{G}_{\mathrm{SRB}}=\mathcal{E}$.

Remark 2. In the case where the pushforward of the Lebesgue measure $\mu$ tends to $\mu_{\mathrm{SRB}}$, one might be tempted to conclude that $\rho(\mu)=\rho\left(\mu_{\mathrm{SRB}}\right)$. This is not necessarily true, and the reason is as follows: suppose $f$ has a Lebesgue measure zero-invariant set $\Lambda$ (such as a horseshoe) away from the support of the SRB measure. The rate at which points escape from a neighborhood of $\Lambda$ will be reflected in $\rho(\mu)$ but not in $\rho\left(\mu_{\mathrm{SRB}}\right)$; this can easily lead to $\rho(\mu)>\rho\left(\mu_{\mathrm{SRB}}\right)$.

III. Restrictions on $\mathcal{G}$ due to the singularities of the map $f$. We state here a version of our results that can be applied to planar billiards; see Theorem F below. Following [KS], we let $U$ be an open smooth (at least $C^{4}$ ) finite-dimensional Riemannian manifold, and assume that $M=\bar{U}$ is a compact metric space of finite capacity $\dagger$, where $\bar{U}$ denotes the closure of $U$.

Let $\iota(x, U)$ be the radius of injectivity of the exponential map $\exp _{x}: T_{x} U \rightarrow U$. We assume that there exist constants $s, c_{0}, \varsigma>0$ such that for each $x, y \in U$, such that $d(x, y)<\iota(x, U)$ and $w=\exp _{x}^{-1}(y)$, we have

$$
\iota(x, U) \geq \min \left\{s, d(x, M \backslash U)^{\varsigma}\right\}, \quad\left\|D\left(\exp _{x}\right)(w)\right\| \leq c_{0} \quad \text { and } \quad\left\|D\left(\exp _{x}^{-1}\right)(y)\right\| \leq c_{0} .
$$

Let $V$ be an open subset of $U$ and let $f: V \rightarrow U$ be a mapping which is a $C^{2}$ diffeomorphism of $V$ onto its image. Let $\mathcal{S}=M \backslash V$. We think of $\mathcal{S}$ as the singularity set of $f$. We assume that there exist constants $C_{1}, a>0$ such that for all $x \in V$,

$$
\left\|D f_{x}\right\| \leq C_{1} d(x, \mathcal{S})^{-a} \text { and }\left\|D f_{x}^{-1}\right\| \leq C_{1} d(x, f \mathcal{S})^{-a} .
$$

Let $\hat{f}_{x}=\exp _{f x}^{-1} \circ f \circ \exp _{x}$ denote the induced map on $T_{x} V$ wherever it is defined. We assume that there exists $b>0$ such that if $x \in V, v \in T_{x} V$ and $\hat{f}_{x}(v)$ is well defined, then

$$
\left\|D^{2} \hat{f}_{x}(v)\right\| \leq C_{1} d\left(\exp _{x}(v), \mathcal{S}\right)^{-b} .
$$

Notice that for billiards with a finite horizon, $a=1$ and $b=3$ (see [CM3, KS]). In what follows, we will assume without loss of generality that $b \geq \varsigma \geq 1$.

Into such a system we introduce a hole $H \subset M$. With regard to the choice of $\mathcal{G}$, in addition to the considerations above, we must also restrict to invariant measures that respect the singularities (see $[\mathbf{K S}]$ ). Define

$$
\mathcal{G}_{\mathcal{S}}=\left\{v \in \mathcal{E} \mid \exists C, \alpha>0 \text { such that for all } \varepsilon>0, v\left(N_{\varepsilon}(\mathcal{S})\right) \leq C \varepsilon^{\alpha}\right\} .
$$

$\dagger$ This means there is some $d<\infty$ such that $\lim _{\sup _{r \rightarrow 0}}(\log C(r) /-\log r)=d$, where $C(r)$ is the minimum cardinality of a covering of $M$ by open balls of radius $r$. For billiards with corners, the set $\bar{U}$ is technically not a manifold with a boundary but a union of such glued together along some boundaries. 


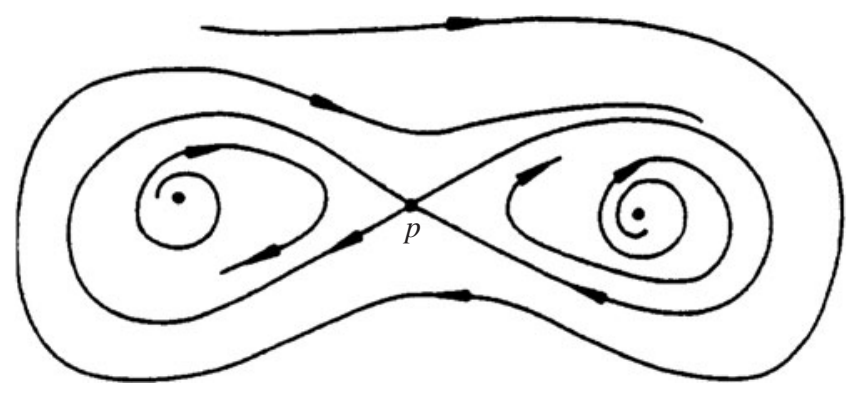

FIGURE 1. Figure 8 attractor. The only invariant measure is $\delta_{p}$, where $p$ is the saddle point.

THEOREM C. Let $(f, M ; H)$ be as above. Then:

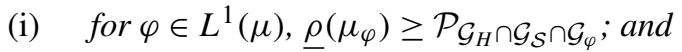

(ii) if $f$ has an $S R \bar{B}$ measure $\mu_{\mathrm{SRB}}$ with no zero Lyapunov exponents, then $\underline{\rho}\left(\mu_{\mathrm{SRB}}\right) \geq$ $\mathcal{P}_{\mathcal{G}_{H} \cap \mathcal{G}_{\mathcal{S}} \cap \mathcal{G}_{\mathrm{SRB}}}$.

We finish with the following.

Remarks on upper bounds and the attractor case. No general results are known for upper bounds on $\rho(m)$, not even for $m=\mu$. Consider the special case where $\Omega \subset M$ is an attractor. Assume there is a neighborhood $O$ of $\Omega$ such that $f(\bar{O}) \subset O$ and $\Omega=\bigcap_{n \geq 0} f^{n}(O)$. Let $H=M \backslash \bar{O}$ and $m=\mu$, so that $\rho(m)=0$ by definition. Since $h_{v}(f) \leq \lambda_{v}^{+}$for all $v \in \mathcal{E}[\mathbf{R}]$, showing that $\rho(m) \leq \mathcal{P}_{\mathcal{G}}$ in this case is equivalent to proving $\mathcal{P}_{\mathcal{E}}=0$. The latter is known to be false in general, an example being the Figure 8 attractor (see Figure 1), so one must rephrase the question to include some notion of 'typicality'. Still, $P_{v}=0$ means either $\lambda_{v}^{+}=0$ or $v$ is an SRB measure [LY], and whether attractors with non-uniform expansion admit SRB measures is well known to be a very difficult question; see e.g. [Y4]. Since any result on upper bounds for $\bar{\rho}(m)$ must include this attractor case, we conclude that in complete generality the question for upper bounds for $\bar{\rho}(m)$ (and lower bounds for escape rates) is intractable at the present time.

We will, however, identify a large class of dynamical systems for which $\underline{\rho}(m)=\bar{\rho}(m)=$ $\mathcal{P}_{\mathcal{G}}$ for some $\mathcal{G}$. This is the content of $\S \S 2.2$ and 2.3.

2.2. Escape rate formula. In this section, we assert for a class of dynamical systems the existence of $\hat{v} \in \mathcal{E}$, the pressure of which is equal to $\rho(m)$, thereby answering (Q1) and (Q2) in the affirmative.

Let $f: M \circlearrowleft$ be a $C^{1+\varepsilon}$ diffeomorphism or a piecewise smooth diffeomorphism, as in the setting of Theorem $\mathrm{C}$, and fix a hole $H \subset M$. We assume:

(A.1) $(f, M)$ has a Markov tower extension $(F, \Delta)$;

(A.2) $(F, \Delta)$ has an exponential tail;

(A.3) $(F, \Delta)$ respects the hole $H$; and

(A.4) the transfer operator on the 'tower with holes' has a spectral gap.

While (A.1) and (A.2) are by now quite standard, and (A.3) and (A.4) have also appeared elsewhere, it will take a few pages to make precise this entire formal setting; we postpone 
that to $\$ 5.1$. Let $\mu_{\mathrm{SRB}}$ denote the (unique) ergodic SRB measure on $\pi(\Delta)$, where $\pi: \Delta \rightarrow M$ is the projection, and let $\mathfrak{r}<1$ be the leading eigenvalue of the transfer operator on the tower with holes.

We will use the following notation: let $m=m^{(0)}$ denote a probability measure on $M$. For $n \geq 1$, let $m^{(n)}$ denote the normalized surviving distribution at time $n$, i.e. $m^{(n)}=$ $f_{*}^{n}\left(\left.m\right|_{M^{n}}\right) / m\left(M^{n}\right)$, assuming $m\left(M^{n}\right)>0$. We call a measure $m$ conditionally invariant with eigenvalue $t$ if $m$ is supported on $M \backslash H$ and $f_{*}\left(\left.m\right|_{M^{1}}\right)=t m$.

THEOREM D. Assume ( $f, M ; H)$ satisfies (A.1)-(A.4). Then:

(a) $\quad \rho\left(\mu_{\mathrm{SRB}}\right)$ is well defined and equals $\log \mathfrak{r}$;

(b) $\mu_{\mathrm{SRB}}^{(n)}$ converges weakly to a conditionally invariant measure $\mu_{*}$ with eigenvalue $\mathfrak{r}$;

(c) there exists $\hat{v} \in \mathcal{G}_{H} \cap \mathcal{G}_{\mathcal{S}}$ such that

$$
\rho\left(\mu_{\mathrm{SRB}}\right)=P_{\hat{v}}:=h_{\hat{v}}(f)-\lambda_{\hat{v}}^{+} ;
$$

(d) $\hat{v}$ is defined by

$$
\hat{v}(\varphi)=\lim _{n \rightarrow \infty} \mathfrak{r}^{-n} \int_{M^{n}} \varphi d \mu_{*} \text { for all continuous } \varphi .
$$

In addition, $\hat{v}$ enjoys exponential decay of correlations on Hölder observables.

Our construction of $\hat{v}$ generalizes that in [CM1, CMS], which assumes the maps in question admit finite Markov partitions. See [BDM] for the first generalization in this direction regarding pressure for one-dimensional maps with holes. Parts (a) and (b) of Theorem D are also known for the periodic Lorentz gas [DWY]. We assert here that these results hold generally for any dynamical system admitting a tower with the stated conditions.

2.3. A full variational principle. Combining the results of the previous two sections, we are able to state a full variational principle (answering (Q1)-(Q3) in §1) for maps admitting towers with a spectral gap, as described in $\$ 2.2$. Let $\Lambda \subset M$ be the reference hyperbolic product set which forms the base of the tower $\Delta$.

Theorem E. Assume ( $f, M ; H)$ satisfies (A.1)-(A.4), and let $\hat{v}$ be as in Theorem $D$.

(a) If $\mu_{\mathrm{SRB}}=\varphi \mu$, where $\varphi \geq \delta>0$ on a neighborhood of $\Lambda$, then $\hat{v} \in \mathcal{G}_{H} \cap \mathcal{G}_{\mathcal{S}} \cap \mathcal{G}_{\varphi}$ and

$$
\rho\left(\mu_{\mathrm{SRB}}\right)=P_{\hat{v}}=\mathcal{P}_{\mathcal{G}_{H} \cap \mathcal{G}_{\mathcal{S}} \cap \mathcal{G}_{\varphi} .}
$$

(b) If $\Lambda$ is contained in a $\mu_{\mathrm{SRB}}$-hyperbolic product set, then $\hat{v} \in \mathcal{G}_{H} \cap \mathcal{G}_{\mathcal{S}} \cap \mathcal{G}_{\mathrm{SRB}}$ and

$$
\rho\left(\mu_{\mathrm{SRB}}\right)=P_{\hat{v}}=\mathcal{P}_{\mathcal{G}_{H} \cap \mathcal{G}_{\mathcal{S}} \cap \mathcal{G}_{\mathrm{SRB}}} .
$$

To our knowledge, the condition in part (b) of Theorem $\mathrm{E}$ can be arranged in all known tower constructions.

Remark on results for tower maps. We will, as an intermediate step to proving Theorems D and E, prove the corresponding results for tower maps with Markov holes. These results are stated as Theorems 4 and 5 in $\$ 5.2$.

An illustrative example: the two-dimensional periodic Lorentz gas. We conclude this section by stating an application of our results to a concrete example. The setting here is 
as in [DWY]: let $f: M \circlearrowleft$ be a billiard map associated with a two-dimensional periodic Lorentz gas with finite horizon whose scatterers are bounded by $C^{3}$ curves with strictly positive curvature. The holes we introduce into $M$ are derived from two types of holes in the billiard table $X$. We say $\sigma \subset X$ is a hole of type $\mathrm{I}$ if $\sigma$ is an open segment of an arc in the boundary of one of the scatterers in $X$. We say $\sigma$ is a hole of type II if it is an open convex set in $X$ whose closure is disjoint from any of the scatterers. The hole $\sigma \subset X$ induces a hole $H_{\sigma} \subset M$, which we also call a hole of type I or type II. See [DWY] for more general holes and details on the geometry they induce in $M$.

THEOREM F. Let $f$ be the billiard map in the last paragraph. Let $H_{\sigma}$ be a hole of type I or type II, and assume it is small enough in the sense of [DWY]. Then:

(a) $\rho\left(\mu_{\mathrm{SRB}}\right)=\mathcal{P}_{\mathcal{G}_{H} \cap \mathcal{G}_{\mathcal{S}}}$; and

(b) there exists $\hat{v} \in \mathcal{G}_{H} \cap \mathcal{G}_{\mathcal{S}}$ such that $P_{\hat{v}}=\rho\left(\mu_{\mathrm{SRB}}\right)$.

Theorem $\mathrm{F}$ is an immediate consequence of Theorems D and $\mathrm{E}$ together with [DWY]: in [DWY], towers with exponential tails respecting arbitrary holes of types I and II are constructed, and for small enough holes the spectral gap property is guaranteed. Thus, the conditions for Theorem D are satisfied; however, [DWY] does not address variational principles or pressure so Theorem D, parts (c) and (d), as well as Theorem F, are new results for this class of billiards.

For the Lorentz gas $\mu_{\mathrm{SRB}}=\varphi \mu$, where $\varphi=c \cos \theta$, so we are in the setting of Theorem E(a); however, $\varphi=0$ only when $\theta= \pm \pi / 2$, so $\mathcal{G}_{\varphi}=\mathcal{E}$ since the set $\{\theta= \pm \pi / 2\}$ does not contain any invariant sets by the finite horizon condition and so cannot contain the support of any invariant measure.

\section{Ideas common to the proofs of Theorems $A-C$}

In this section, we first give the ideas common to the proofs of Theorems A-C. Let $f$ be the mapping in question, let $m$ be the reference measure (i.e. $m=\mu_{\varphi}$ in Theorem A, $m=\mu_{\text {SRB }}$ in Theorem B, and so on), and let $\mathcal{G}$ be the relevant set of ergodic invariant measures with respect to which the pressure term is defined (i.e. $\mathcal{G}=\mathcal{G}_{H} \cap \mathcal{G}_{\varphi}$ in Theorem A, and so on). This 'generic' notation is used throughout $\S 3$.

If $\mathcal{G}=\emptyset$, then $\mathcal{P}_{\mathcal{G}}=-\infty$ and the theorem is vacuously true. Consider $v \in \mathcal{G}$. Leaving precision for later, our proof will proceed as follows: for $n \geq 0$, we introduce dynamical balls in $M^{n}$ of the form

$$
B(x, n, g)=\left\{y \in M: d\left(f^{i} x, f^{i} y\right)<g\left(f^{i} x\right), 0 \leq i \leq n\right\} \cap M^{n},
$$

where $M^{n}=\bigcap_{i=0}^{n} f^{-i}(M \backslash H)$ and $g: M \rightarrow \mathbb{R}^{+}$is a suitable function to be specified (think of it as $g \approx \varepsilon$ for the moment). We will prove the following.

I. Relation to entropy. $v(B(x, n, g)) \sim e^{-n h_{v}(f)}$.

II. Volume estimate. $m(B(x, n, g)) \gtrsim e^{-n \lambda_{\nu}^{+}}$, where $\lambda_{v}^{+}$is the sum of positive Lyapunov exponents for $v$-a.e. $x$.

From estimate (I), we deduce that $M^{n}$ contains $\gtrsim e^{n h_{v}(f)}$ disjoint sets of the type $B(x, n, g)$. This, together with estimate (II), gives

$$
m\left(M^{n}\right) \gtrsim e^{n h_{v}(f)} \cdot e^{-n \lambda_{v}^{+}} .
$$


Taking log, dividing by $n$ and letting $n \rightarrow \infty$, gives $\underline{\rho}(m) \geq h_{v}(f)-\lambda_{v}^{+}$, which is what we need.

We now proceed to make these ideas precise.

I. Relation to entropy. For this part we cite the following very general result.

Proposition 3.1. Let $\Phi: X \circlearrowleft$ be a measurable transformation of a compact metric space of finite capacity, and let $\theta$ be an ergodic invariant measure for $\Phi$. Let $\hat{g}_{\varepsilon}$ be a family of functions satisfying $\left|\hat{g}_{\varepsilon}\right|_{\infty} \leq \varepsilon$ and $\int_{X}-\log \hat{g}_{\varepsilon} d \theta<\infty$, and define

$$
\hat{B}\left(x, n, \hat{g}_{\varepsilon}\right)=\left\{y \in X: d\left(\Phi^{i} x, \Phi^{i} y\right)<\hat{g}_{\varepsilon}\left(\Phi^{i} x\right), 0 \leq i \leq n\right\} .
$$

Then, for $\theta$-a.e. $x$,

$$
\lim _{\varepsilon \rightarrow 0^{+}} \liminf _{n \rightarrow \infty}-\frac{1}{n} \log \theta\left(\hat{B}\left(x, n, \hat{g}_{\varepsilon}\right)\right)=\lim _{\varepsilon \rightarrow 0^{+}} \limsup _{n \rightarrow \infty}-\frac{1}{n} \log \theta\left(\hat{B}\left(x, n, \hat{g}_{\varepsilon}\right)\right)=h_{\theta}(\Phi) .
$$

Proposition 3.1 follows from [M, Lemma 2] and [BK, Main theorem]. Note that although $[\mathbf{B K}]$ is phrased in terms of a continuous map, the proof does not use this fact.

In the proofs of Theorems $\mathrm{A}-\mathrm{C}$, Proposition 3.1 will be applied with $\Phi=f, \theta=v \in \mathcal{G}$ and

$$
\hat{g}_{\varepsilon}(x):=\min \{\varepsilon, d(x, \mathcal{S})\}
$$

$\left(\mathcal{S}=\emptyset\right.$ in Theorems A and B). Observe that intersecting $\hat{B}\left(x, n, \hat{g}_{\varepsilon}\right)$ with $M^{n}$ does not affect its $\nu$-measure since $v$ is supported on the survivor set. From $v\left(N_{\varepsilon}(\mathcal{S})\right) \leq C \varepsilon^{\alpha}$, we have

$$
\begin{aligned}
& \int_{M}-\log \left(\hat{g}_{\varepsilon}\right) d v \leq-\log \varepsilon+\sum_{n=0}^{\infty} v\left(N_{\varepsilon e^{-n}}(\mathcal{S}) \backslash N_{\varepsilon e^{-(n+1)}}(\mathcal{S})\right)(n+1-\log \varepsilon) \\
& \leq-\log \varepsilon+\sum_{n=0}^{\infty} C \varepsilon^{\alpha} e^{-\alpha n}(n+1-\log \varepsilon)<\infty,
\end{aligned}
$$

so our $\hat{g}_{\varepsilon}$ satisfies the hypotheses of Proposition 3.1.

II. Volume estimate. Let $g_{\varepsilon}=\frac{1}{3} \hat{g}_{\varepsilon}$. Continuing to let $m$ denote the initial distribution and $v \in \mathcal{G}$, we state the following desired volume estimate.

PROPOSITION 3.2. There exists a measurable set $E \subset \Omega$ with $v(E)>0$ such that for $v$ a.e. $x \in E$,

$$
\sup _{\varepsilon>0} \limsup _{n \rightarrow \infty}-\frac{1}{n} \log m\left(B\left(x, n, g_{\varepsilon}\right)\right) \leq \lambda_{\nu}^{+}
$$

Proof of Theorems A-C assuming Proposition 3.2. Let $v \in \mathcal{G}$ be given. We fix $\delta>0$, and let $\sigma:=v(E)$, where $E$ is as in Proposition 3.2. Using Propositions 3.1 and 3.2, we may choose first $\varepsilon>0$ sufficiently small, and then $n_{0}=n_{0}(\delta, \varepsilon) \in \mathbb{Z}^{+}$sufficiently large, and a measurable set $E^{\prime} \subset E$ with $v\left(E^{\prime}\right) \geq \sigma / 2$ such that for every $x \in E^{\prime}$ :

(i) $\quad v\left(B\left(x, n, 3 g_{\varepsilon}\right)\right) \leq e^{-n\left(h_{\nu}-\delta\right)}$ for all $n \geq n_{0}$; and

(ii) $m\left(B\left(x, n, g_{\varepsilon}\right)\right) \geq e^{-n\left(\lambda_{v}^{+}+\delta\right)}$ for all $n \geq n_{0}$. 
For $n \geq n_{0}$, let $\mathcal{C}_{n} \subset E^{\prime}$ be a maximal set of points such that $B\left(x_{i}, n, g_{\varepsilon}\right) \cap$ $B\left(x_{j}, n, g_{\varepsilon}\right)=\emptyset$ whenever $x_{i}, x_{j} \in \mathcal{C}_{n}, x_{i} \neq x_{j}$. By the maximality of $\mathcal{C}_{n}$, for every $y \in E^{\prime}$, there exists $x_{i} \in \mathcal{C}_{n}$ such that $B\left(y, n, g_{\varepsilon}\right) \cap B\left(x_{i}, n, g_{\varepsilon}\right) \neq \emptyset$. We will show momentarily that $y \in B\left(x_{i}, n, 3 g_{\varepsilon}\right)$. This will imply $E^{\prime} \subset \bigcup_{x_{i} \in \mathcal{C}_{n}} B\left(x_{i}, n, 3 g_{\varepsilon}\right)$, and hence $\left|\mathcal{C}_{n}\right| \geq(\sigma / 2) e^{n\left(h_{\mu}-\delta\right)}$ by (i).

To show $y \in B\left(x_{i}, n, 3 g_{\varepsilon}\right)$, it suffices to show $d\left(f^{k} y, f^{k} x_{i}\right)<3 g_{\varepsilon}\left(f^{k} x_{i}\right)$ for all $k \leq n$ since $y \in E^{\prime} \subset M^{n}$. Now, $B\left(y, n, g_{\varepsilon}\right) \cap B\left(x_{i}, n, g_{\varepsilon}\right) \neq \emptyset$ means there exists $z \in M$ such that $d\left(f^{k} x_{i}, f^{k} z\right) \leq g_{\varepsilon}\left(f^{k} x_{i}\right)$ and $d\left(f^{k} z, f^{k} y\right) \leq g_{\varepsilon}\left(f^{k} y\right)$ for all $0 \leq k \leq n$. Thus, the assertion above boils down to the following lemma.

LeMmA 3.3. For any $x, y \in M$, if there exists $z \in M$ with $d(x, z) \leq g_{\varepsilon}(x)$ and $d(z, y) \leq$ $g_{\varepsilon}(y)$, then $d(x, y) \leq 3 g_{\varepsilon}(x)$.

Proof of lemma. It suffices to show $g_{\varepsilon}(y) \leq 2 g_{\varepsilon}(x)$, for that will imply

$$
d(x, y) \leq d(x, z)+d(z, y) \leq g_{\varepsilon}(x)+g_{\varepsilon}(y) \leq 3 g_{\varepsilon}(x),
$$

proving the lemma. Observe that

$$
\begin{aligned}
d(y, \mathcal{S}) & \leq d(y, z)+d(z, x)+d(x, \mathcal{S}) \\
& \leq g_{\varepsilon}(y)+g_{\varepsilon}(x)+d(x, \mathcal{S}) \leq \frac{1}{3} d(y, \mathcal{S})+\frac{4}{3} d(x, \mathcal{S})
\end{aligned}
$$

the last inequality following from $g_{\varepsilon}(\cdot) \leq \frac{1}{3} d(\cdot, \mathcal{S})$. Altogether, this gives $d(y, \mathcal{S}) \leq$ $2 d(x, \mathcal{S})$.

To finish, consider the following two cases.

Case 1: $d(x, \mathcal{S})>\varepsilon$. With $g_{\varepsilon}(x)=\frac{1}{3} \varepsilon, g_{\varepsilon}(y)$ is automatically $<2 g_{\varepsilon}(x)$ since it is $\leq \frac{1}{3} \varepsilon$.

Case 2: $d(x, \mathcal{S}) \leq \varepsilon$. In this case, $g_{\varepsilon}(y) \leq \frac{1}{3} d(y, \mathcal{S}) \leq \frac{2}{3} d(x, \mathcal{S})=2 g_{\varepsilon}(x)$.

For each $x \in E^{\prime}$, we have $B\left(x, n, g_{\varepsilon}\right) \subset M^{n}$ by definition. Since the $B\left(x_{i}, n, g_{\varepsilon}\right)$ are disjoint, we may estimate $m\left(M^{n}\right)$ by

$$
m\left(M^{n}\right) \geq \sum_{x_{i} \in \mathcal{C}_{n}} m\left(B\left(x_{i}, n, g_{\varepsilon}\right)\right) \geq\left|\mathcal{C}_{n}\right| \cdot \min _{x_{i} \in \mathcal{C}_{n}} m\left(B\left(x_{i}, n, g_{\varepsilon}\right)\right) \geq \frac{\sigma}{2} e^{n\left(h_{v}-\delta\right)} e^{-n\left(\lambda_{v}^{+}+\delta\right)} .
$$

This yields

$$
\liminf _{n \rightarrow \infty} \frac{1}{n} \log m\left(M^{n}\right) \geq h_{v}(f)-\lambda_{v}^{+}-2 \delta
$$

The theorem is proved since $\delta$ was chosen arbitrarily.

To complete the proofs of Theorems A-C, it remains only to prove the volume estimate in Proposition 3.2.

\section{Volume estimates}

In this section, we prove Proposition 3.2 in the various settings of interest. The basic argument, which treats the case $\mathcal{S}=\emptyset, m=\mu$, and $v \in \mathcal{G}_{H}$, is presented in $\S 4$.1. Proofs of other cases in Theorems A-C are presented as modifications of this one.

4.1. Proof of Proposition 3.2: basic setup. We consider here the most basic setup, namely where $\mathcal{S}=\emptyset, m=\mu$, and $\nu \in \mathcal{G}_{H}$ (as defined), and give a proof of Proposition 3.2. 
I. Plan. From the pointwise nature of the result and the fact that the quantity on the left of (7) increases as $\varepsilon \rightarrow 0$, it suffices to show that, given $\kappa>0$, for $v$-a.e. $x$ and arbitrarily small $\varepsilon>0$, there exists $c(x, \varepsilon)$ such that

$$
m\left(B\left(x, n, g_{\varepsilon}\right)\right) \geq c(x, \varepsilon) e^{-n\left(\lambda_{v}^{+}+\kappa\right)} \quad \text { for all } n \geq 0 .
$$

Here, $g_{\varepsilon}(\cdot) \equiv \frac{1}{3} \varepsilon$; remember that $B\left(x, n, g_{\varepsilon}\right)$ is a dynamical ball in $M^{n}$ (and not in $M$ ). Such an object is cumbersome to work with since it involves both the dynamics and the hole. To remove the hole from consideration, we introduce

$$
B^{*}(x, n, \varepsilon, \gamma):=\left\{y \in M: d\left(f^{i} x, f^{i} y\right)<\varepsilon e^{-\gamma i} \text { for } 0 \leq i<n\right\} .
$$

By definition of $\mathcal{G}_{H}$, for any $\gamma>0$ and v-a.e. $x, B^{*}\left(x, n, \frac{1}{3} \varepsilon, \gamma\right) \subset B\left(x, n, g_{\varepsilon}\right)$ for small enough $\varepsilon$. Thus, it suffices to prove, for a suitably chosen $\gamma$ and arbitrarily small $\varepsilon>0$,

$$
m\left(B^{*}(x, n, \varepsilon, \gamma)\right) \geq c(x, \varepsilon, \gamma) e^{-n\left(\lambda_{v}^{+}+\kappa\right)} \quad \text { for all } n \geq 0 .
$$

This is what we will do. Our strategy is to make these volume estimates in Lyapunov charts and pass them back to the manifold.

II. Lyapunov charts and hyperbolic estimates. Let $\lambda_{1}<\cdots<\lambda_{p}$ be the distinct Lyapunov exponents of $(f, v)$, with multiplicities $m_{1}, \ldots, m_{p}$ respectively, and let $E_{i}(x)$ be the subspace of $T_{x} M$ corresponding to $\lambda_{i}$. For each $i$, we let $R_{i}(r)$ denote the ball of radius $r$ centered at 0 in $\mathbb{R}^{m_{i}}$, and let $R(r)=\prod_{i=1}^{p} R_{i}(r)$. We recall below the following facts about Lyapunov charts, following the exposition in [Y2].

Proposition 4.1. [Y2, §3.1] Let $\delta \ll \min _{i \neq j}\left|\lambda_{i}-\lambda_{j}\right|$ be fixed. Then there is a measurable set $V^{\prime} \subset M, v\left(V^{\prime}\right)=1$, a measurable function $\ell: V^{\prime} \rightarrow[1, \infty)$ satisfying $\ell\left(f^{ \pm} x\right) / \ell(x)<e^{2 \delta}$, and a family of charts $\left\{\Phi_{x}: R\left(\delta \ell(x)^{-1}\right) \rightarrow M\right\}_{x \in V^{\prime}}$ with the following properties:

(a) (i) $\Phi_{x}(0)=x$;

(ii) $D \Phi_{x}\left(\{0\} \times \cdots \times \mathbb{R}^{m_{i}} \times \cdots \times\{0\}\right)=E_{i}(x)$; and

(iii) for all $z, z^{\prime} \in R\left(\delta \ell(x)^{-1}\right)$,

$$
K^{-1} d\left(\Phi_{x} z, \Phi_{x} z^{\prime}\right) \leq\left|z-z^{\prime}\right| \leq \ell(x) d\left(\Phi_{x} z, \Phi_{x} z^{\prime}\right),
$$

where $K$ is a constant depending only on the dimension of $M$.

(b) Let $\tilde{f}_{x}=\Phi_{f x}^{-1} \circ f \circ \Phi_{x}$ be defined where it makes sense. Then:

(i) $\quad e^{\lambda_{i}-\delta}|v| \leq\left|D \tilde{f}_{x}(0) v\right| \leq e^{\lambda_{i}+\delta}|v|$ for $v \in\{0\} \times \cdots \times \mathbb{R}^{m_{i}} \times \cdots \times\{0\}$;

(ii) $\operatorname{Lip}\left(\tilde{f}_{x}-D \tilde{f}_{x}(0)\right)<\delta$; and

(iii) $\operatorname{Lip}\left(D \tilde{f}_{x}\right)<\ell(x)$.

The following notation is used: let $T_{x} M=E^{c u}(x) \oplus E^{s}(x)$, where $E^{c u}(x)=$ $\bigoplus_{i: \lambda_{i} \geq 0} E_{i}(x)$ and $E^{s}(x)=\bigoplus_{i: \lambda_{i}<0} E_{i}(x)$. We will estimate the volume of the sets in question by looking at slices parallel to $E^{c u}$, and will do so in Lyapunov charts. Let $R^{c u}$ and $R^{s}$ be the subspaces in the charts corresponding to $E^{c u}$ and $E^{s}$, and let $R^{c u}(r)$ and $R^{s}(r)$ denote disks of radius $r$ centered at 0 in $R^{c u}$ and $R^{s}$, respectively. We will work with compositions of chart maps, writing

$$
\tilde{f}_{x}^{n}:=\tilde{f}_{f^{n-1} x} \circ \cdots \circ \tilde{f}_{x}
$$

and study graph transforms by $\tilde{f}_{x}^{n}$ of functions from $R^{c u}(r)$ to $R^{s}(r)$. The precise assertions are as follows. 
(a) For all $\gamma>0$ sufficiently small, there exist $\delta, \sigma>0$ small enough and a chart system (with $\delta$ as in Proposition 4.1) such that the following holds for $v$-a.e. $x$ : let $r \leq \delta \ell(x)^{-1}$, and let $g_{0}: R^{c u}(r) \rightarrow R^{s}(r)$ be a $C^{1}$ function with $\left|g_{0}(0)\right|<\frac{1}{2} r$ and $\left\|D g_{0}\right\|<\sigma$. Then, for $i=1,2, \ldots$, , there exists $g_{i}: R^{c u}\left(e^{-\gamma i} r\right) \rightarrow R^{s}\left(e^{-\gamma i} r\right)$ defined on exponentially shrinking domains and with $\left\|D g_{i}\right\|<\sigma$ for all $i$ such that, inductively,

$$
\tilde{f}_{f^{i-1} x}\left(\operatorname{graph}\left(g_{i-1}\right)\right) \cap R\left(e^{-\gamma i} r\right)=\operatorname{graph}\left(g_{i}\right) .
$$

That is to say, if $\Gamma_{z}$ is the graph transform by $\tilde{f}_{z}$, then for each $i, \Gamma_{f^{i-1} x}\left(g_{i-1}\right)=g_{i}$.

(b) For $g: R^{c u}(r) \rightarrow R^{s}(r)$ and $y \in \operatorname{graph}(g)$, let $T_{g}(y)$ denote the tangent space to the graph of $g$ at $y$. If $\delta$ and $\sigma$ in (a) are small enough, then for $y \in \operatorname{graph}\left(g_{0}\right)$ such that $\tilde{f}_{x}^{i}(y) \in R\left(e^{-\gamma i} r\right)$ for all $i \leq n$,

$$
\left|\operatorname{det}\left[\left.D \tilde{f}_{x}^{n}(y)\right|_{T_{g_{0}}(y)}\right]\right|<e^{n\left(\lambda^{+}+3 k \delta\right)},
$$

where $\lambda^{+}=\sum_{i: \lambda_{i}>0} m_{i} \lambda_{i}$ and $k=\operatorname{dim}\left(E^{c u}\right)$.

Notice first that with $2 \delta<\gamma$, we are assured that $R\left(e^{-\gamma i} r\right)$ lies in the chart at $f^{i} x$; this is because $\ell\left(f^{i} x\right)>e^{-\gamma i} \ell(x)$; see Proposition 4.1. Since most of the other assertions in (a) and (b) follow from standard (uniformly hyperbolic) graph transform estimates, we will only sketch the arguments for a few key points. (A version of these estimates can be found in [Y2, §3.1]; see also [Y1, §B] for similar results.)

The 'overflowing property' of the graph transforms can be justified as follows. Consider first the case where $g_{0}(0)=0$. By Proposition 4.1(b)(i), $\left|D \tilde{f}_{x}(0) v\right| \geq e^{-\delta}|v| \approx(1-\delta)|v|$ for $v \in R^{c u}$. By Proposition 4.1(b)(iii) together with the chart size, we have, for all $\eta \in R(r)$

$$
\left|D \tilde{f}_{x}(\eta) v-D \tilde{f}_{x}(0) v\right| \leq \operatorname{Lip}\left(D \tilde{f}_{x}\right) r|v|<\delta|v| .
$$

This gives $\left|D \tilde{f}_{x}(\eta) v\right|>(1-3 \delta)|v| \approx e^{-3 \delta}|v|$ for $v$ with a small enough component in $R^{s}$. Thus, with $\delta$ and $\sigma$ sufficiently small relative to $\gamma$, the overflowing property is assured from step to step for $g_{0}$ with $g_{0}(0)=0$. For graphs that do not pass through 0 , we pivot them at $y \in \tilde{W}_{\text {loc }}^{s} \cap \operatorname{graph}\left(g_{0}\right)$, where $\tilde{W}_{\text {loc }}^{s}$ is the stable manifold of $x$ in its chart. Since $\left|\tilde{f}_{x}^{i}(y)\right|<e^{\left(\lambda_{s}+\delta\right) i}$, where $\lambda_{s}=\max \left\{\lambda_{i}: \lambda_{i}<0\right\}$, movements of $\tilde{f}_{x}^{i}(y)$ in the $R^{c u}$-direction are negligible, assuming $\gamma \ll\left|\lambda_{s}\right|$.

The assertion in (b) is proved similarly: we view $\operatorname{det}\left(D \tilde{f}_{x}^{n}\right)$ as a product of determinants. At each step, $\left|\operatorname{det}\left(\left.D \tilde{f}_{f^{i} x}(0)\right|_{R^{c u}}\right)\right|<e^{\lambda^{+}+k \delta}$, and we may assume that approximations of the type in the last paragraph increase the error by a factor $<e^{2 k \delta}$.

III. Completing the proof. Putting assertions (a) and (b) in II together, we arrive at the following: define

$$
\tilde{B}^{*}(x, n, r, \gamma)=\left\{y \in R^{c u}(r) \times R^{s}\left(\frac{1}{2} r\right): \tilde{f}_{x}^{i}(y) \in R\left(e^{-\gamma i} r\right) \text { for } i=1,2, \ldots, n\right\} .
$$

We foliate $\tilde{B}^{*}(x, 0, r, \gamma)$ with planes $\{P\}$ parallel to $R^{c u}(r) \times\{0\}$, and view them as graphs of constant functions. By the overflowing property of the graph transform at each step, $\tilde{f}_{x}^{i}(y) \in R\left(e^{-\gamma i} r\right)$ for all $i \leq n$ is equivalent to $\tilde{f}_{x}^{n}(y) \in R\left(e^{-\gamma n} r\right)$. Pulling back $\tilde{f}_{x}^{n}(P) \cap R\left(e^{-\gamma n} r\right)$, we use the bound in assertion (b) to estimate the area of $P \cap \tilde{B}^{*}(x, n, r, \gamma)$. We then integrate over $\{P\}$ to obtain

$$
\operatorname{Leb}\left(\tilde{B}^{*}(x, n, r, \gamma)\right) \geq\left(\frac{r}{2}\right)^{d-k} \cdot\left(r e^{-\gamma n}\right)^{k} \cdot e^{-n\left(\lambda^{+}+3 k \delta\right)},
$$

where $d=\operatorname{dim}(M)$. 
We now return to the argument outlined at the beginning of the proof. Let $\kappa>0$ be given. Assuming always $\gamma \ll\left|\lambda_{s}\right|$, we now take it small enough that $4 k \gamma<\kappa$, and let $\delta$ be small enough (with respect to $\gamma$ ) for assertions (a) and (b) to hold in the chart system $\left\{\Phi_{x}\right\}$ associated with $\delta$. For $\nu$-typical $x$, we consider $\varepsilon$ small enough that $B\left(f^{i} x, \varepsilon e^{-\gamma i}\right) \cap$ $H=\emptyset$ for all $i \geq 0$. Choosing $r<\varepsilon / K$, where $K$ is as in Proposition 4.1(a)(iii), we define $\tilde{B}^{*}(x, n, r, \gamma)$ in the chart at $x$ as above, and observe that $\Phi_{x}\left(\tilde{B}^{*}(x, n, r, \gamma)\right) \subset$ $B^{*}(x, n, \varepsilon, \gamma)$. To finish, it remains to pass the estimate in (9) back to $M$. Proposition 4.1(a)(iii) gives a bound on the Jacobian of $\Phi_{x}$, allowing us to conclude that

$$
m\left(\Phi_{x}\left(\tilde{B}^{*}(x, n, r, \gamma)\right)\right) \geq \ell(x)^{-d} \cdot \operatorname{Leb}\left(\tilde{B}^{*}(x, n, r, \gamma)\right) \geq c e^{-n\left(\lambda^{+}+\kappa\right)}
$$

for some constant $c$ depending on $x, \varepsilon$ and $\gamma$.

4.2. Adaptations of basic argument to various settings. We now explain how each of the other results in Theorems $\mathrm{A}-\mathrm{C}$ is deduced from the proof in $\S 4.1$.

1. The $W^{s}$-neighborhood condition $(O)$. We continue to assume $\mathcal{S}=\emptyset$ and $m=\mu$. To relax the condition from the original definition of $\mathcal{G}_{H}$ in $\$ 2.1$ to the one given by $(\mathrm{O})$, the proof in $\S 4.1$ is modified as follows: given $\kappa$, we fix $\gamma, \delta$, a chart system $\left\{\Phi_{x}\right\}$, and a $\nu$ typical $x \in M$. Let $O$, a $W^{s}$-neighborhood, and $\varepsilon$ be such that $f^{i}(O) \cap B\left(f^{i} x, \varepsilon e^{-\gamma i}\right) \subset$ $M \backslash H$ for all $i$. We need to show $m\left(O \cap B^{*}(x, n, \varepsilon, \gamma)\right) \geq c(x, \varepsilon, \gamma) e^{-n\left(\lambda_{v}^{+}+\kappa\right)}$. Let $r<\varepsilon / K$.

The following notation is used: for $y \in R(r)$ and small $\eta>0$, let $R(y, \eta)=y+$ $R(\eta)$; if $y=\left(y^{c u}, y^{s}\right)$ are the coordinates of $y$ with respect to $R^{c u}$ and $R^{s}$, we write $R^{c u}\left(y^{c u}, \eta\right)=y^{c u}+R^{c u}(\eta)$, and so on. To define the analog of $\tilde{B}^{*}(x, n, r, \gamma)$ in $\S 4.1$, let $z \in O \cap W_{\text {loc }}^{s}(x)$ be sufficiently close to $x$, let $\tilde{z}:=\Phi_{x}^{-1}(z)$, and let $r^{\prime}<r$ be small enough that $\Phi_{x}\left(R\left(\tilde{z}, r^{\prime}\right)\right) \subset O$. Define

$$
\begin{aligned}
& \tilde{B}^{*}\left(x, n, z, r^{\prime}, \gamma\right) \\
& \quad:=\left\{y \in R^{c u}\left(\tilde{z}^{c u}, r^{\prime}\right) \times R^{s}\left(\tilde{z}^{s}, \frac{1}{2} r^{\prime}\right): \tilde{f}_{x}^{i}(y) \in R\left(\tilde{f}_{x}^{i}(\tilde{z}), e^{-\gamma i} r^{\prime}\right) \text { for } i=1,2, \ldots, n\right\} .
\end{aligned}
$$

Since $z \in W_{\mathrm{loc}}^{s}(x), \tilde{f}_{x}^{i}(\tilde{z}) \rightarrow 0$ as $i \rightarrow \infty$. It is straightforward to check that modulo a constant, $\operatorname{Leb}\left(\tilde{B}^{*}\left(x, n, z, r^{\prime}, \gamma\right)\right)$ is bounded below by the quantity on the right side of (9), and that $\Phi_{x}\left(\tilde{B}^{*}\left(x, n, z, r^{\prime}, \gamma\right)\right) \subset\left(O \cap B^{*}(x, n, \varepsilon, \gamma)\right)$.

In the settings below, we will revert back to $\mathcal{G}_{H}$ as defined, leaving it to the reader to extend the proof to include the condition $(\mathrm{O})$ if they so choose.

2. Initial distributions with densities. Continuing to assume $\mathcal{S}=\emptyset$, we let $m=\mu_{\varphi}$ for some $\varphi \in L^{1}(\mu)$. Let $v \in \mathcal{G}_{H} \cap \mathcal{G}_{\varphi}$, and let $Z$ and $c_{\nu}$ have the meaning in the definition of $\mathcal{G}_{\varphi}$. Observe that for $v$-a.e. $x \in Z$ and small enough $\varepsilon$, one has $\mu_{\varphi}\left(B^{*}(x, n, \varepsilon, \gamma)\right) \geq$ $c_{\nu} m\left(B^{*}(x, n, \varepsilon, \gamma)\right)$. An argument identical to that in $\S 4.1$ proves Proposition 3.2 with $E=Z$.

3. SRB measures as initial distributions. Continuing to assume $\mathcal{S}=\emptyset$, we let $m=$ $\mu_{\mathrm{SRB}}$, as in Theorem B. Given $v \in \mathcal{G}_{H} \cap \mathcal{G}_{\mathrm{SRB}}$, we fix a $\mu_{\mathrm{SRB}}$ hyperbolic product set $\Pi=\left(\bigcup \Gamma^{u}\right) \cap\left(\bigcup \Gamma^{s}\right)$ with $v(\Pi)>0$, and show that the volume estimate for $m\left(B^{*}(x, n, \varepsilon, \gamma)\right)$ in $\S 4.1$ holds for $\nu$-a.e. $x \in \Pi$. 
Let $x \in \omega_{x}^{u} \cap \omega_{x}^{s} \in \Pi$ be a $\nu$-typical point, where $\omega_{x}^{u} \in \Gamma^{u}$ and $\omega_{x}^{s} \in \Gamma^{s}$. Note that as a result of the uniform contraction of $T^{n} \omega_{x}^{s}$ and $T^{-n} \omega_{x}^{u}$ required by (W.1) of $\$ 2.1(\mathrm{~B}), x$ can have no zero Lyapunov exponents. Let $\tilde{W}_{\text {loc }}^{u}$ denote the image of the local unstable manifold through $x$ in its chart. Since local unstable manifolds are unique (up to size), $\Phi_{x}^{-1}\left(\omega_{x}^{u}\right) \subset \tilde{W}_{\text {loc }}^{u}$, which has the dimension of $R^{u}$ and is tangent to it at 0 . (Since no zero Lyapunov exponents is an assumption for Theorem $\mathrm{B}$, we have $R^{u}$ instead of $R^{c u}$.) By conditions (W.1) and (W.2), for all small enough $r>0$, there exists $\Gamma_{0} \subset \Gamma^{u}$ such that (i) $\mu_{\mathrm{SRB}}\left(\bigcup_{\omega \in \Gamma_{0}} \omega\right)>0$ and (ii) for every $\omega \in \Gamma_{0}, \Phi_{x}^{-1}(\omega) \cap R(r)$ is the graph of a function from $R^{u}(r)$ to $R^{s}(r)$ with the properties of $g_{0}$ in $\S 4$.1. Define

$$
\tilde{B}^{*}\left(x, n, r, \Gamma_{0}, \gamma\right)=\left\{y \in \bigcup_{\omega \in \Gamma_{0}} \Phi_{x}^{-1} \omega: \tilde{f}_{x}^{i}(y) \in R\left(e^{-\gamma i} r\right) \text { for } i=0,1,2, \ldots, n\right\} .
$$

With $r$ small enough relative to $\varepsilon$, clearly $\Phi_{x}\left(\tilde{B}^{*}\left(x, n, r, \Gamma_{0}, \gamma\right)\right) \subset B^{*}(x, n, \varepsilon, \gamma)$. To estimate the measure of this set, it is more convenient to bring $\mu_{\mathrm{SRB}}$ to the chart (instead of doing it on $M)$ : let $\alpha$ be the measure $\left(\Phi_{x}^{-1}\right)_{*}\left(\left.\mu_{\mathrm{SRB}}\right|_{\cup_{\omega \in \Gamma_{0}}} \omega\right)$ restricted to $R(r)$. By (i) above together with (W.3), $\alpha\left(\tilde{B}^{*}\left(x, 0, r, \Gamma_{0}, \gamma\right)\right)>0$. We disintegrate $\alpha$ into conditional probability measures on the leaves $\left\{\Phi_{x}^{-1} \omega\right\}$, letting $\alpha_{T}$ denote the measure in the transverse direction. To estimate the $\alpha$-measure of $\tilde{B}^{*}\left(x, n, r, \Gamma_{0}, \gamma\right)$, we do it one $\Phi_{x}^{-1} \omega$-leaf at a time, integrating with respect to $\alpha_{T}$ afterwards. Condition (W.3) ensures uniform lower bounds of the type in (9) for $\alpha_{T}$-almost all leaves.

4. Maps with singularities. We discuss the case $m=\mu$, leaving the others to the reader.

Let $v \in \mathcal{G}_{H} \cap \mathcal{G}_{\mathcal{S}}$, and observe the following lemma.

LEMmA 4.2. Let $E_{\varepsilon, \gamma}=\left\{x \in M: d\left(f^{i} x, \mathcal{S}\right)>\varepsilon e^{-\gamma i}\right.$ for all $\left.i \geq 0\right\}$. Then, for any fixed $\gamma>0, \lim _{\varepsilon \rightarrow 0} v\left(E_{\varepsilon, \gamma}\right)=1$.

Proof. This follows from the simple estimate

$$
\nu\left(M \backslash E_{\varepsilon, \gamma}\right)=\sum_{i \geq 0} \nu\left[f^{-i}\left(N_{\varepsilon e^{-\gamma i}}(\mathcal{S})\right)\right]=\sum_{i \geq 0} \nu\left[N_{\varepsilon e^{-\gamma i}}(\mathcal{S})\right] \leq \sum_{i \geq 0} C \varepsilon^{\alpha} e^{-\gamma \alpha i} \leq C^{\prime} \varepsilon^{\alpha},
$$

the first inequality coming from the definition of $\mathcal{G}_{\mathcal{S}}$. (See also [KS, Part I, Lemma 3.1].)

This means that for $x \in E_{\varepsilon, \gamma}$, we again have $B^{*}\left(x, n, \frac{1}{3} \varepsilon, \gamma\right) \subset B\left(x, n, g_{\varepsilon}\right)$, for $g_{\varepsilon}\left(f^{i} x\right)=\frac{1}{3} \min \left\{\varepsilon, d\left(f^{i} x, \mathcal{S}\right)\right\} \geq \frac{1}{3} \varepsilon e^{-\gamma i}$.

Continuing to follow the proof in $\S 4.1$, we note that the definition of $\mathcal{G}_{\mathcal{S}}$, together with (3), implies that $\int_{M} \log ^{+}\left\|D f_{x}^{ \pm 1}\right\| d v<\infty$, where $\log ^{+} x=\max \{\log x, 0\}$, so the Lyapunov exponents are well defined $v$-almost everywhere. In addition, the Lyapunov charts described in Proposition 4.1 exist for this class of maps with some modifications because of the presence of singularities.

Observe first that (a)(i), (a)(ii) and (b)(i) of Proposition 4.1 hold as stated since these quantities depend only on $D f$ at a typical point $x$ (see [KS, Part I, Theorem 2.2]) $\dagger$.

$\dagger$ Although [KS] uses only a single splitting, $T_{x} M=E_{\alpha}(x) \oplus E_{\beta}(x)$, one can just as easily split the tangent space into $\bigoplus_{i} E_{i}(x)$, one for each Lyapunov exponent, to obtain Proposition 4.1(b)(i) using an argument identical to that in [Y2, §3.1]. 
The other items of Proposition 4.1 are modified as follows. Fix $\delta$ as in Proposition 4.1. Then there exist a set $V^{\prime}$ with $v\left(V^{\prime}\right)=1$ and a measurable function $\ell(x): V^{\prime} \rightarrow[1, \infty)$, with $\ell\left(f^{ \pm} x\right)<e^{2 \delta} \ell(x)$, such that for all $\varepsilon>0$ sufficiently small, the charts $\Phi_{x}$ are defined on $R\left(\delta \ell(x)^{-1} g_{\varepsilon}(x)^{b}\right)$, where $b$ is the exponent from (4), and satisfy the following.

(a) (iii') For all $z, z^{\prime} \in R\left(\delta \ell(x)^{-1} g_{\varepsilon}(x)^{b}\right)$,

$$
K^{-1} d\left(\Phi_{x} z, \Phi_{x} z^{\prime}\right) \leq\left|z-z^{\prime}\right| \leq \ell(x) d\left(\Phi_{x} z, \Phi_{x} z^{\prime}\right),
$$

where $K$ is a constant depending only on the dimension of $M$ and $c_{0}$ from (2).

(b) Let $\tilde{f}_{x}=\Phi_{f x}^{-1} \circ f \circ \Phi_{x}$ be defined where it makes sense. Then:

(ii') $\operatorname{Lip}\left(\tilde{f}_{x}-D \tilde{f}_{x}(0)\right) \leq \delta$; and

(iii') $\operatorname{Lip}\left(D \tilde{f}_{x}\right) \leq \ell(x) g_{\varepsilon}(x)^{-b}$.

Although the construction of these charts is similar to that found in [KS, P], we include the necessary arguments in the Appendix since the statements we need are somewhat different from those found in the literature.

With the charts $\left\{\Phi_{x}\right\}$ in place, the proof follows a similar line to that given in $\S 4.1$, with slight modifications because of the singularities. For example, assertion (a) is no longer a uniform statement for all $x \in V^{\prime}$; rather, we need to choose $r \leq \delta \ell(x)^{-1} g_{\varepsilon}(x)^{b}$, but only after $\varepsilon$ is fixed depending on the rate of approach of $x$ to the singularities. We state precisely these changes below.

Fix $\kappa>0$ and choose $\gamma \ll\left|\lambda_{s}\right|$ such that $(b+4) k \gamma<\kappa$. Using Lemma 4.2, we choose $\varepsilon>0$ such that $v\left(E_{\varepsilon, \gamma}\right)>1-\kappa$. Next we choose $\delta>0$ with $2 \delta<\gamma$, so that there exists a chart system $\left\{\Phi_{x}\right\}_{x \in V^{\prime}}$ with the modified properties as listed in (a)(iii')-(b)(iii') above. Note that $v\left(V^{\prime} \cap E_{\varepsilon, \gamma}\right)>1-\kappa$.

We now choose $x \in V^{\prime} \cap E_{\varepsilon, \gamma}$ and prove estimate (8). Note that $B\left(f^{i} x, \varepsilon e^{-\gamma i}\right) \cap(H \cup$ $\mathcal{S})=\emptyset$. Finally, choosing $r \leq \delta \ell(x)^{-1} g_{\varepsilon}(x)^{b}$ guarantees that the assertions (a) and (b) of $\$ 4.1$ hold along the orbit of $x$ with $\gamma$ replaced by $\gamma(b+1)$, for then $R\left(r e^{-i \gamma(b+1)}\right)$ lies in the chart at $f^{i} x$ by definition of $E_{\varepsilon, \gamma}$ and choice of $r$. In particular, $\tilde{f}_{f^{i} x}$ is defined on $R\left(r e^{-i \gamma(b+1)}\right)$. We shrink $r$ further if necessary so that $r<\varepsilon /(3 K)$ and define $\tilde{B}^{*}(x, n, r, \gamma(b+1))$ as in $\S 4.1$. Then, by item (a) (iii') above, $\Phi_{x}\left(\tilde{B}^{*}(x, n, r, \gamma(b+\right.$ 1)) $) \subset B^{*}\left(x, n, \frac{1}{3} \varepsilon, \gamma\right)$ and the rest of the proof follows line by line with only minor changes to constants. For example, (9) has the factor $\left(r e^{-\gamma n(b+1)}\right)^{k}$, as indicated above.

This proves Proposition 3.2 for all $x \in E_{\varepsilon, \gamma}$. But since $\kappa>0$ was chosen arbitrarily, by Lemma 4.2, we conclude that Proposition 3.2 holds for $v$-a.e. $x$.

\section{Towers with holes}

This section is exclusively about escape dynamics on towers. Section 5.1 reviews basic facts and notation for towers, making precise (A.1)-(A.4) in $\$ 2.2$. In $\$ 5.2$, we formulate results analogous to Theorems D and E for towers with Markov holes. Proofs are given in $\S \S 5.3$ and 5.4 .

\subsection{Review of definitions and basic facts}

I. Closed systems (without holes). Let $f: M \circlearrowleft$ be a (piecewise) $C^{1+\epsilon}$ diffeomorphism. The material below is taken from [Y3]. We recall only essential definitions, referring the reader to [Y3] for details. 
Generalized horseshoes. The idea of a generalized horseshoe with infinitely many branches and variable return times, denoted $(\Lambda, R)$, is as follows: $\Lambda \subset M$ is a compact subset with a hyperbolic product structure, i.e. $\Lambda=\left(\bigcup \Gamma^{u}\right) \cap\left(\bigcup \Gamma^{s}\right)$, where $\Gamma^{s}$ and $\Gamma^{u}$ are continuous families of local stable and unstable manifolds, and $\mu_{\omega}\{\omega \cap \Lambda\}>0$ for every $\omega \in \Gamma^{u}$, where $\mu_{\omega}$ is the Riemannian measure on the unstable manifold $\omega$. We say $\Lambda^{s}$ is an $s$-subset of $\Lambda$ if $\Lambda^{s}=\left(\bigcup \Gamma^{u}\right) \cap\left(\bigcup \tilde{\Gamma}^{s}\right)$ for some $\tilde{\Gamma}^{s} \subset \Gamma^{s}$, and $u$-subsets are defined similarly. Modulo a set the restriction of which to each $\omega \in \Gamma^{u}$ has $\mu_{\omega}$-measure $0, \Lambda$ is a countable disjoint union of (closed) $s$-subsets $\Lambda_{j}$ with the property that for each $j$, there exists $R_{j} \in \mathbb{Z}^{+}$such that $f^{R_{j}}\left(\Lambda_{j}\right)$ is a $u$-subset of $\Lambda$. The function $R: \Lambda \rightarrow \mathbb{Z}^{+}$ given by $\left.R\right|_{\Lambda_{j}}=R_{j}$ is called the return time function to $\Lambda$.

The definition of a generalized horseshoe includes conditions on hyperbolicity formulated as (P1)-(P5) in [Y3]. We will omit them and focus instead on the estimates derived from these conditions that we will need. Let $\omega^{s}(x)$ and $\omega^{u}(x)$ denote, respectively, the elements of $\Gamma^{s}$ and $\Gamma^{u}$ containing $x$.

- $\quad$ There is a separation time $s: \Lambda \rightarrow \mathbb{Z}^{+}$with the properties that:

(i) $s(x, y)=s\left(x^{\prime}, y^{\prime}\right)$ for $x^{\prime} \in \omega^{s}(x), y^{\prime} \in \omega^{s}(y)$;

(ii) for $x, y \in \Lambda_{j}, s(x, y) \geq R_{j}$; and

(iii) for $x \in \Lambda_{j}, y \in \Lambda_{j^{\prime}}, j \neq j^{\prime}, s(x, y) \leq \min \left(R_{j}, R_{j^{\prime}}\right)$.

- There are constants $C>0$ and $\alpha \in(0,1)$, related to the hyperbolicity and distortion of $f$, such that if $y \in \omega^{s}(x)$, then $d\left(f^{n} x, f^{n} y\right) \leq C \alpha^{n}$ for all $n \geq 0$.

The following facts about the Jacobian in the unstable direction are useful. For $\omega, \omega^{\prime} \in$ $\Gamma^{u}$, the holonomy map $\Theta_{\omega, \omega^{\prime}}: \omega \cap \Lambda \rightarrow \omega^{\prime} \cap \Lambda$ is obtained by sliding along stable curves, i.e. $\Theta_{\omega, \omega^{\prime}}(x)=\omega^{s}(x) \cap \omega^{\prime}$. Fix an arbitrary leaf $\hat{\omega} \in \Gamma^{u}$. We let $\hat{\Theta}(x)$ be the unique point in $\omega^{s}(x) \cap \hat{\omega}$, and define $a(x)=\log \prod_{i=0}^{\infty}\left(\operatorname{det} D f^{u}\left(f^{i} x\right) / \operatorname{det} D f^{u}\left(f^{i}(\hat{\Theta} x)\right)\right)$, where $\operatorname{det} D f^{u}(x)=\operatorname{det}\left(\left.D f(x)\right|_{E^{u}(x)}\right)$ is the unstable Jacobian of $f$. This function is used to define a family of reference measures $\left\{m_{\omega}, \omega \in \Gamma^{u}\right\}$, where $m_{\omega}$ is the measure on $\omega$ whose density with respect to $\mu_{\omega}$ is $e^{a} \cdot 1_{\omega \cap \Lambda}$. For $x \in \omega \cap \Lambda_{i}$, let $\omega^{\prime}$ be such that $f^{R_{i}}\left(\omega \cap \Lambda_{i}\right)=\omega^{\prime}$. We define $J^{u}\left(f^{R}\right)(x)=J_{m_{\omega}, m_{\omega^{\prime}}}\left(f^{R_{i}} \mid\left(\omega \cap \Lambda_{i}\right)\right)(x)$, the Jacobian of $f^{R}$ with respect to the measures $m_{\omega}$ and $m_{\omega^{\prime}}$.

Remark on notation. It is convenient in this section to follow the notation in [Y3], some of which conflicts, however, with earlier notation. For example, $m$ in the last paragraph is not intended to signify any relation to initial distributions in escape dynamics, and $C_{1}$ below is not related to the same notation in $\$ 2.1$, paragraph III. We do not believe this will lead to problems as the contexts are quite different.

LEMMA 5.1. [Y3, Lemma 1]

(a) For all $\omega, \omega^{\prime} \in \Gamma^{u},\left(\Theta_{\omega, \omega^{\prime}}\right)_{*} m_{\omega}=m_{\omega^{\prime}}$.

(b) For each $\omega \in \Gamma^{u}$ and $x \in \omega, J^{u}\left(f^{R}\right)(x)=J^{u}\left(f^{R}\right)(y)$ for all $y \in \omega^{s}(x)$.

(c) There exists $C_{1}>0$ (depending on $C$ and $\alpha$ ) such that for each $\omega \in \Gamma^{u}, i \in \mathbb{Z}^{+}$and all $x, y \in \Lambda_{i} \cap \omega$,

$$
\left|\frac{J^{u}\left(f^{R}\right)(x)}{J^{u}\left(f^{R}\right)(y)}-1\right| \leq C_{1} \alpha^{s\left(f^{R} x, f^{R} y\right) / 2} .
$$

(d) $\sup _{x \in \Lambda} a(x)<\infty$ and $|a(x)-a(y)| \leq 4 C \alpha^{\frac{1}{2} s(x, y)}$ on each $\omega \in \Gamma^{u}$. 
We say $(\Lambda, R)$ has exponential return times if there exist $C_{0}>0$ and $\theta_{0}>0$ such that for all $\omega \in \Gamma^{u}, \mu_{\omega}\{R>n\} \leq C_{0} \theta_{0}^{n}$ for all $n \geq 0$. This property (in fact, integrability of $R$ is sufficient) plus the requirement that g.c.d. $\{R\}=1$ guarantees that $f$ has a unique SRB measure $\mu_{\mathrm{SRB}}$ with $\mu_{\mathrm{SRB}}(\Lambda)>0$ [Y3, Theorem 1].

'Hyperbolic' Markov towers. Given $f$ with a generalized horseshoe $(\Lambda, R)$, it is shown in [Y3] that one can associate a Markov extension $F: \Delta \rightarrow \Delta$, which focuses on the return dynamics, to $\Lambda$. The set $\Delta$ is the disjoint union $\bigcup_{\ell>0} \Delta_{\ell}$, where $\Delta_{\ell}$, the $\ell$ th level of the tower, is defined to be $\Delta_{\ell}=\{(x, \ell): x \in \Lambda, R(x)>\ell\}$, and $F$ is defined by $F(x, \ell)=(x, \ell+1)$ for $\ell<R(x)-1$ and $F(x, \ell)=\left(f^{R} x, 0\right)$ when $\ell=R(x)-1$; that is to say, $F$ maps $(x, 0)$ successively up the tower until the return time for $x$ is reached. A projection $\pi: \Delta \rightarrow M$ with $\pi \circ F=f \circ \pi$ is uniquely defined, assuming the natural identification of $\Delta_{0}$ with $\Lambda$.

For notational simplicity, we will often refer to a point in $\Delta$ as $x$ when the level $\ell$ is made clear by context.

The separation function $s(\cdot, \cdot)$ above defines a countable partition $\left\{\Delta_{\ell, j}\right\}$ on $\Delta$ : for $x, y \in \Delta_{0}, s(x, y)=\inf \left\{n>0: F^{n} x, F^{n} y\right.$ lie in different $\left.\Delta_{\ell, j}\right\}$. It is easy to see that $\left\{\Delta_{\ell, j}\right\}$ is a Markov partition for $F$ with $\Delta_{0}$ as a single element. Let $\Delta_{\ell, j}^{*}=\Delta_{\ell, j} \cap F^{-1} \Delta_{0}$. Note that $\left.F\right|_{\Delta_{\ell, j}^{*}}$ maps $\Delta_{\ell, j}^{*}$ bijectively onto a $u$-subset of $\Delta_{0}$, and if we rename the collection $\left\{F^{-\ell} \Delta_{\ell, j}^{*}\right\}$ as $\left\{\left(\Delta_{0}\right)_{i}\right\}$, then $\left\{\left(\Delta_{0}\right)_{i}\right\}$ is a countable collection of closed subsets of $\Delta_{0}$, the $\pi$-images of which are precisely the $\left\{\Lambda_{i}\right\}$ in the paragraph on generalized horseshoes.

Stable and unstable sets for $\Delta_{\ell, j}$ are defined as follows: let $\Gamma^{s}\left(\pi\left(\Delta_{\ell, j}\right)\right)$ and $\Gamma^{u}\left(\pi\left(\Delta_{\ell, j}\right)\right)$ be the stable and unstable families defining the hyperbolic product set $\pi\left(\Delta_{\ell, j}\right)$. We say $\tilde{\omega} \subset \Delta_{\ell, j}$ is an unstable set of $\Delta_{\ell, j}$ if $\pi(\tilde{\omega})=\omega \cap \pi\left(\Delta_{\ell, j}\right)$ for some $\omega \in \Gamma^{u}\left(\pi\left(\Delta_{\ell, j}\right)\right)$. Since there can be no ambiguity, we will use $\Gamma^{u}\left(\Delta_{\ell, j}\right)$ to denote the set of all such $\tilde{\omega}$, and let $\Gamma^{u}(\Delta)=\bigcup_{\ell, j} \Gamma^{u}\left(\Delta_{\ell, j}\right)$. Stable sets of $\Delta_{\ell, j}$ and $\Gamma^{s}(\Delta)$ are defined similarly.

Two reference measures $\tilde{\mu}_{\omega}$ and $\tilde{m}_{\omega}$ are defined on $\omega \in \Gamma^{u}(\Delta)$ as follows: on $\Delta_{0}$, identifying $\omega \in \Gamma^{u}\left(\Delta_{0}\right)$ with $\omega^{\prime} \cap \Lambda$ for $\omega^{\prime} \in \Gamma^{u}(\Lambda), \tilde{\mu}_{\omega}$ is simply $\left.\mu_{\omega^{\prime}}\right|_{\omega^{\prime} \cap \Lambda}$ and $\tilde{m}_{\omega}$ is $m_{\omega^{\prime}}$. Once these measures are defined on $\omega \in \Gamma^{u}\left(\Delta_{0}\right)$, there is exactly one way to extend them to $\bigcup_{\ell>0} \Gamma^{u}\left(\Delta_{\ell}\right)$ so that if $J_{\mu}^{u}(F)$ and $J^{u}(F)$ denote the Jacobians of $F$ on unstable sets with respect to $\tilde{\mu}_{\omega}$ and $\tilde{m}_{\omega}$, respectively, then $J^{u}(F)=J_{\mu}^{u}(F)=1$ on $\Delta \backslash F^{-1}\left(\Delta_{0}\right)$. Notice also that if we extend $a$ to $\bigcup_{\ell>0} \Delta_{\ell}$ by $a(x)=a\left(F^{-1} x\right)$, then $d \tilde{m}_{\omega}=e^{a} d \tilde{\mu}_{\omega}$ on all $\omega \in \Gamma^{u}(\Delta)$.

Quotient 'expanding' towers. Associated with $F: \Delta \rightarrow \Delta$ is a quotient tower $\bar{F}: \bar{\Delta} \rightarrow$ $\bar{\Delta}$ obtained by collapsing stable sets to points, i.e. $\bar{\Delta}=\Delta / \sim$, where, for $x, y \in \Delta, x \sim y$ if and only if $y \in \omega(x)$ for some $\omega \in \Gamma^{S}(\Delta)$. Let $\bar{\pi}: \Delta \rightarrow \bar{\Delta}$ be the projection defined by $\sim$. We will use the notation $\bar{\Delta}_{\ell}=\bar{\pi}\left(\Delta_{\ell}\right), \bar{\Delta}_{\ell, j}=\bar{\pi}\left(\Delta_{\ell, j}\right)$, and so on.

Lemmas 5.1(a) and (b) together imply that there is a natural measure $\bar{m}$ on $\bar{\Delta}$ with respect to which the Jacobian of $\bar{F}, J \bar{F}$, is well defined: specifically, we have $J \bar{F} \equiv 1$ on $\bar{\Delta} \backslash \bar{F}^{-1}\left(\bar{\Delta}_{0}\right)$, and for $x \in \bar{\Delta}_{0}, J \bar{F}^{R}(x)=J^{u}\left(f^{R}\right)(y)$ for any $y \in \omega^{s}(x)$. Finally, with the definition of separation time inherited from $\Delta_{0}$, the distortion bound in Lemma 5.1(c) holds for $J \bar{F}^{R}$ on $\bar{\Delta}_{0}$. 
II. Systems with holes. The setting is as in paragraph I. We fix an open set $H \subset M$ and call it 'the hole'.

Towers with Markov holes (following [DWY]). Let $(F, \Delta)$ be the tower arising from the horseshoe $(\Lambda, R)$. We say $(F, \Delta)$ respects the hole $H$ if the following conditions are satisfied:

(H.1) $\pi^{-1} H$ is the union of countably many elements of $\left\{\Delta_{\ell, j}\right\}$; and

(H.2) $\pi\left(\Delta_{0}\right) \subset M \backslash H$, and there exist $\delta>0, \xi_{1}>1$ such that all $x \in \pi\left(\Delta_{0}\right)$ satisfy $d\left(f^{n} x, \mathcal{S} \cup \partial H\right) \geq \delta \xi_{1}^{-n}$ for all $n \geq 0$.

Because of (H.1), we refer to $\pi^{-1} H$, the hole on $\Delta$, as a 'Markov hole'. This implies in particular that for every $i$ and $\ell$ with $0 \leq \ell<R_{i}, f^{\ell}\left(\Lambda_{i}\right)$ either does not meet $H$ or it is completely contained in $H$. Equivalently, on the tower $(F, \Delta)$, each $\left(\Delta_{0}\right)_{i}$ either falls into the hole completely on its way up the tower or returns to $\Delta_{0}$ intact.

Earlier on we used $(f, M ; H)$ to denote an open system. Observe that $\left(F, \Delta ; \pi^{-1} H\right)$ and $(\bar{F}, \bar{\Delta} ; \bar{H})$, where $\bar{H}=\bar{\pi}\left(\pi^{-1} H\right)$, are open systems of the same type. As before, we write

$$
\Delta^{n}=\bigcap_{i=0}^{n} F^{-i}\left(\Delta \backslash \pi^{-1} H\right)=\left\{x \in \Delta: F^{i} x \notin \pi^{-1} H \text { for } 0 \leq i \leq n\right\},
$$

and $\Delta^{\infty}=\bigcap_{i=0}^{\infty} \Delta^{n}$. In particular, $\Delta^{0}=\Delta \backslash \pi^{-1} H$. The notation $\stackrel{\circ}{F}^{n}=\left.F^{n}\right|_{\Delta^{n}}$ for $n \geq 1$ is sometimes used to distinguish between systems with and without holes. Corresponding objects for $(\bar{F}, \bar{\Delta} ; \bar{H})$ are denoted by $\bar{\Delta}^{n}$ and $\bar{\Delta}^{\infty}$, etc.

III. Abstract towers and the notion of spectral gap. In paragraphs I and II, we considered towers that arise from generalized horseshoes. Towers can, in fact, be defined in the abstract. Leaving details to the reader, an abstract expanding tower is a dynamical system $\bar{F}: \bar{\Delta} \rightarrow \bar{\Delta}$, where $\bar{\Delta}_{0}$ is a compact set, $\bar{\Delta}=\bigcup_{\ell \geq 0} \bar{\Delta}_{\ell}$ has a tower structure, $\bar{F}$ moves points up the tower until their return time $R$; there is a countable Markov partition $\left\{\bar{\Delta}_{\ell, j}\right\}$ on $\bar{\Delta}$ which is a generator and a reference measure $\bar{m}$ with respect to which we have (i) $J \bar{F}=1$ on $\bar{\Delta} \backslash \bar{F}^{-1}\left(\bar{\Delta}_{0}\right)$ and (ii) modulo a set of $\bar{m}$-measure zero, $\bar{\Delta}_{0}=\bigcup_{i}\left(\bar{\Delta}_{0}\right)_{i}$, where $\bar{F}^{R}$ maps each closed set $\left(\bar{\Delta}_{0}\right)_{i}$ homeomorphically onto $\bar{\Delta}_{0}$ with the distortion bound in Lemma 5.1(c). Abstract expanding towers with Markov holes $\bar{H}$ are defined in the obvious way, as are abstract hyperbolic towers.

Given $(\bar{F}, \bar{\Delta})$ with $\bar{m}\{R>n\}<C_{0} \theta_{0}^{n}$ for some $C_{0} \geq 1$ and $\theta_{0}<1 \dagger$, we fix $\beta$ with $1>\beta>\max \left\{\theta_{0}, \sqrt{\alpha}\right\}$, where $\alpha$ is as in Lemma $5.1(\mathrm{c})$, and define a symbolic metric on $\bar{\Delta}$ by $d_{\beta}(x, y)=\beta^{s(x, y)}$. Since $\beta>\sqrt{\alpha}$, Lemma 5.1(c) implies that $J \bar{F}$ is logLipshitz with respect to this metric. Let $\mathcal{B}=\left\{\psi \in L^{1}(\bar{\Delta}, \bar{m}):\|\psi\|<\infty\right\}$, where $\|\psi\|=$ $\|\psi\|_{\infty}+\|\psi\|_{\text {Lip }}$ and

$$
\|\psi\|_{\infty}=\sup _{\ell, j} \sup _{x \in \bar{\Delta}_{\ell, j}}|\psi(x)| \beta^{\ell}, \quad\|\psi\|_{\operatorname{Lip}}=\sup _{\ell, j} \operatorname{Lip}\left(\left.\psi\right|_{\bar{\Delta}_{\ell, j}}\right) \beta^{\ell} .
$$

$\operatorname{Lip}(\cdot)$ in the last displayed formula is with respect to the symbolic metric $d_{\beta}$, and $(\mathcal{B},\|\cdot\|)$ so defined is a Banach space.

$\dagger$ Our default rule is to use the same symbol for corresponding objects for $f, F$ and $\bar{F}$ when no ambiguity can arise, given context. Thus, $R$ is the name of the return time function on $\Lambda, \Delta_{0}$ and $\bar{\Delta}_{0}$. 
Now consider the open system $(\bar{F}, \bar{\Delta} ; \bar{H})$, where $\bar{H}$ is a Markov hole. Following [BDM], we let $\overline{\mathcal{L}}$ denote the transfer operator associated with $\left.\bar{F}\right|_{\bar{\Delta}^{1}}$ defined on $\mathcal{B}$, i.e. for $\psi \in \mathcal{B}$ and $x \in \bar{\Delta}$,

$$
\overline{\mathcal{L}} \psi(x)=1_{\bar{\Delta}^{0}}(x) \sum_{y \in \bar{\Delta}^{0} \cap \bar{F}^{-1}\{x\}} \psi(y)(J \bar{F}(y))^{-1} .
$$

We say $(\bar{F}, \bar{\Delta} ; \bar{H})$ has a spectral gap if:

(i) $\overline{\mathcal{L}}$ is quasi-compact with a unique eigenvalue $\mathfrak{r}$ of maximum modulus; and

(ii) $\mathfrak{r}$ is real with $\beta<\mathfrak{r}<1$; it is simple, with a one-dimensional eigenspace.

Notice that if $h \in \mathcal{B}$ satisfies $\overline{\mathcal{L}} h=\mathfrak{r} h$, then $h \bar{m}$ defines a conditionally invariant measure for $\bar{F}$ with eigenvalue r, i.e. $\left.\bar{F}_{*}(h \bar{m})\right|_{\bar{\Delta} \backslash \bar{H}}=\mathfrak{r} \cdot h \bar{m}$.

Finally, if $(F, \Delta)$ is an abstract hyperbolic tower that projects onto $(\bar{F}, \bar{\Delta})$, and $\tilde{H} \subset \Delta$ is a Markov hole which projects onto $\bar{H}$, then we say $(F, \Delta ; \tilde{H})$ has a spectral gap if $(\bar{F}, \bar{\Delta} ; \bar{H})$ does.

The conditions (A.1)-(A.4) in $\$ 2.2$ have now been made precise.

5.2. Variational principles for $(\bar{F}, \bar{\Delta} ; \bar{H})$ and $\left(F, \Delta ; \pi^{-1} H\right)$. As noted earlier, our aim in this section is to prove, as an intermediate step for Theorems D and E, a version of the corresponding results for the open system $\left(F, \Delta ; \pi^{-1} H\right)$. These results are deduced from some previously known results for $(\bar{F}, \bar{\Delta} ; \bar{H})$, which we first recall.

I. Results for expanding towers. We consider here an abstract expanding tower $(\bar{F}, \bar{\Delta} ; \bar{H})$ with Markov holes. The following notation is used: let $\mathcal{B}$ be the function space above, and define $\mathcal{B}_{0}$ to be the set of bounded functions in $\mathcal{B}$ whose Lipschitz constant is also bounded, i.e. the definition of $\mathcal{B}_{0}$ is the same as that of $\mathcal{B}$, but with the weights $\beta^{\ell}$ removed. Let $\mathcal{M}_{\bar{F}}\left(\bar{\Delta}^{\infty}\right)$ denote the set of invariant measures on $\bar{\Delta}^{\infty}$, and define

$$
\mathcal{G}_{\bar{\Delta}}=\left\{\bar{\eta} \in \mathcal{M}_{\bar{F}}\left(\bar{\Delta}^{\infty}\right) \mid \bar{\eta}(\log J \bar{F})<\infty\right\} .
$$

THEOREM 3. (Mostly [BDM]; see remark below) Assume $\bar{m}\{R>n\}<C_{0} \theta_{0}^{n}$, and $(\bar{F}, \bar{\Delta} ; \bar{H})$ has a spectral gap with largest eigenvalue $\mathfrak{r}$. Let $h_{*} \in \mathcal{B}$ be the unique eigenfunction of $\mathfrak{r}$ with $\int h_{*} d \bar{m}=1$. Then:

(a) there exist constants $D>0$ and $\tau<1$ such that for all $\psi \in \mathcal{B}$,

$$
\left\|\mathfrak{r}^{-n} \overline{\mathcal{L}}^{n} \psi-d(\psi) h_{*}\right\| \leq D\|\psi\| \tau^{n}, \quad \text { where } d(\psi)=\lim _{n \rightarrow \infty} \mathfrak{r}^{-n} \int_{\bar{\Delta}^{n}} \psi d \bar{m}<\infty .
$$

Assume additionally that:

(*) there exist $\bar{C}>0$ and $\bar{\theta} \in\left(\mathfrak{r}^{-1} \theta_{0}, 1\right)$ such that $\left.\log J \bar{F}^{n}\right|_{\bar{\Delta}_{0} \cap\{R=n\}} \leq \bar{C}^{-n}$ for all $n \geq 0$;

(b) $\log \mathfrak{r}=\mathcal{P}_{\mathcal{G}_{\bar{\Delta}}}:=\sup _{\bar{\eta} \in \mathcal{G}_{\bar{\Delta}}}\left\{h_{\bar{\eta}}(\bar{F})-\int_{\bar{\Delta}} \log J \bar{F} d \bar{\eta}\right\}$;

(c) let $\bar{v}$ be defined by

$$
\bar{v}(\varphi)=\lim _{n \rightarrow \infty} \mathfrak{r}^{-n} \int_{\bar{\Delta}^{n}} \varphi h_{*} d \bar{m} \quad \text { for all } \varphi \in \mathcal{B}_{0} .
$$

Then $\bar{v} \in \mathcal{G}_{\bar{\Delta}}$ and attains the supremum in $(b)$; 
(d) other properties of $\bar{v}$ are that $(\bar{F}, \bar{v})$ is ergodic, and enjoys exponential decay of correlations between $\varphi$ and $\psi \circ \bar{F}^{n}$ for $\varphi \in \mathcal{B}_{0}$ and $\psi \in L^{\infty}$.

Remark. The restriction $\bar{\eta}(\log J \bar{F})<\infty$, which appears in the definition of $\mathcal{G}_{\bar{\Delta}}$, is omitted in $[\mathbf{B D M}]$, as is the condition $(*)$, which is extremely mild $\dagger$, but a condition of this type is needed to ensure that $\bar{v} \in \mathcal{G}_{\bar{\Delta}}$. Since a main novelty of Theorem 3 is the non-compactness of the phase space $\bar{\Delta}$, and these conditions are directly connected to the finiteness of various quantities, we will provide sketches of corrected proofs of Theorem 3(b) and (c) in $\$ 5.3$. The proofs of parts (a) and (d) in [BDM] are unaffected.

II. Results for hyperbolic towers arising from $(f, M ; H)$. We now return to the setting of $\S 2.1$, where $f: M \circlearrowleft$ is a $C^{1+\epsilon}$ diffeomorphism with or without singularities. Let $H \subset M$, and assume that the open system $(f, M ; H)$ satisfies (A.1)-(A.4) in $\$ 2.1$.

We first recall the following result proved in [DWY] as part of our study of billiard systems with holes. Let $\tilde{\mathcal{B}}$ be the class of measures $\sigma$ on $\Delta$ with the following properties.

(i) $\sigma$ has absolutely continuous conditional measures on unstable leaves.

(ii) $\bar{\pi}_{*} \sigma=\bar{\psi}_{\sigma} d \bar{m}$ for some $\bar{\psi}_{\sigma} \in \mathcal{B}$.

THEOREM 4. [DWY] Under the conditions above, the following hold for $\left(F, \Delta ; \pi^{-1} H\right)$. (a) For all $\sigma \in \tilde{\mathcal{B}}$ with $d\left(\bar{\psi}_{\sigma}\right)>0$, where $d\left(\bar{\psi}_{\sigma}\right)>0$ is as in Theorem 3(a),

$$
\log \mathfrak{r}=\lim _{n \rightarrow \infty} \frac{1}{n} \log \sigma\left(\Delta^{n}\right) \text { i.e. } \rho(\sigma)=\log \mathfrak{r} .
$$

(b) There exists a conditionally invariant distribution $\tilde{\mu}_{*} \in \tilde{\mathcal{B}}$ such that $\stackrel{\circ}{*}_{*} \tilde{\mu}_{*}=\mathfrak{r} \tilde{\mu}_{*}$, $\bar{\pi}_{*} \tilde{\mu}_{*}=h_{*} \bar{m}$, and for which the following hold: for all $\sigma \in \tilde{\mathcal{B}}$,

$$
\lim _{n \rightarrow \infty} \mathfrak{r}^{-n} \stackrel{\circ}{F}_{*}^{n} \sigma=d\left(\bar{\psi}_{\sigma}\right) \cdot \tilde{\mu}_{*} \text {, and if } d\left(\bar{\psi}_{\sigma}\right)>0 \text {, then } \lim _{n \rightarrow \infty} \frac{\stackrel{\circ}{F}_{*}^{n} \sigma}{\dot{\circ}_{*}^{n} \sigma(\Delta)}=\tilde{\mu}_{*},
$$

where the convergence is in the weak* topology.

The measure $\tilde{\mu}_{*}$ can be thought of as the physical measure for the leaky system $\left(F, \Delta ; \pi^{-1} H\right)$.

We formulate in Theorem 5 the results which, along with Theorem 4 , will give the analogs of Theorems $\mathrm{D}$ and $\mathrm{E}$ for $\left(F, \Delta ; \pi^{-1} H\right)$. Let $\mathcal{M}_{F}\left(\Delta^{\infty}\right)$ denote the set of invariant probability measures supported on $\Delta^{\infty}$, and define

$$
\mathcal{G}_{\Delta}=\left\{\eta \in \mathcal{M}_{F}\left(\Delta^{\infty}\right) \mid \eta\left(\log J_{\mu}^{u} F\right)<\infty\right\}
$$

Furthermore, let $C_{b}^{0}(\Delta)$ be the set of bounded functions on $\Delta$ which are continuous on each $\Delta_{\ell, j}$. We postpone the definitions of $\operatorname{Lip}^{s}(\Delta)$ and $\operatorname{Lip}^{u}(\Delta)$ (other function spaces that will appear) until after the theorem.

THEOREM 5. Let $\left(F, \Delta ; \pi^{-1} H\right)$ be as above. Then the following hold.

(a) $\log \mathfrak{r}=\mathcal{P}_{\mathcal{G}_{\Delta}}=\sup _{\eta \in \mathcal{G}_{\Delta}}\left\{h_{\eta}(F)-\int_{\Delta} \log J_{\mu}^{u} F d \eta\right\}$.

$\dagger$ We observe that $(*)$ holds for all the towers constructed in [BDM]; indeed, in that setting, $\left.\log J \bar{F}^{n}\right|_{\bar{\Delta}_{0} \cap\{R=n\}} \leq$ $C n$ and all measures $\bar{\eta} \in \mathcal{M}_{\bar{F}}\left(\bar{\Delta}^{\infty}\right)$ satisfy $\bar{\eta}(\log J \bar{F})<\infty$. 
(b) Let $\tilde{v}$ be defined by

$$
\tilde{v}(\varphi)=\lim _{n \rightarrow \infty} \mathfrak{r}^{-n} \int_{\Delta^{n}} \varphi d \tilde{\mu}_{*} \quad \text { for all } \varphi \in C_{b}^{0}(\Delta) .
$$

Then $\tilde{v} \in \mathcal{G}_{\Delta}$ and it attains the supremum in $(a)$.

(c) Other properties of $\tilde{v}$ are that $(F, \tilde{v})$ is ergodic, and exhibits exponential decay of correlations between $\varphi$ and $\psi \circ F^{n}$ for $\varphi \in \operatorname{Lip}^{u}(\Delta)$ and $\psi \in \operatorname{Lip}^{s}(\Delta)$.

The function spaces $\operatorname{Lip}^{s}(\Delta)$ and $\operatorname{Lip}^{u}(\Delta)$ are defined as follows. For $\omega^{s} \in \Gamma^{s}(\Delta)$ and $x, y \in \omega^{s} \subset \Delta_{0}$, we denote by $d_{s}(x, y)$ the distance between $\pi(x)$ and $\pi(y)$, according to the Riemannian metric on $M$, and extend $d_{s}$ to $\omega^{s} \in \bigcup_{\ell>0} \Gamma^{s}\left(\Delta_{\ell}\right)$ by setting $d_{s}\left(F^{\ell} x, F^{\ell} y\right)=\alpha^{\ell} d_{s}(x, y)$ for all $\ell<R(x)$ and $y \in \omega^{s}(x)$. It then follows from \$5.1.I that $d_{s}\left(F^{n} x, F^{n} y\right) \leq C \alpha^{n}$ for all $n \geq 0$ and $x, y \in \Delta, y \in \omega^{s}(x)$. For $\varphi \in C_{b}^{0}$, let $|\varphi|_{\text {Lip }}^{s}$ be the supremum of Lipshitz constants of $\left.\varphi\right|_{\omega^{s}}$ with respect to $d_{s}$, as $\omega^{s}$ ranges over all stable sets in $\Gamma^{s}(\Delta)$. Then $\operatorname{Lip}^{s}(\Delta)=\left\{\varphi \in C_{b}^{0}:|\varphi|_{\text {Lip }}^{s}<\infty\right\}$. The function space $\operatorname{Lip}^{u}(\Delta)$ is defined similarly using $|\varphi|_{\text {Lip }}^{u}$, where $|\varphi|_{\text {Lip }}^{u}$ is the Lipschitz constant of $\varphi$ restricted to unstable sets in the metric $d_{\beta}$.

5.3. Outline of proof of Theorem 3(b), (c): [BDM] amended. We assume part (a) of Theorem 3 has been proved, and proceed to the proofs of parts (b) and (c), following mostly $[\mathbf{B D M}]$ and highlighting several finiteness issues.

1. Return map to $\bar{\Delta}_{0}^{\infty}$ and the full shift $T: \Sigma_{\infty} \circlearrowleft$. Since $\bar{F}$ is not defined everywhere on $\bar{\Delta}$, let us first make precise the definition of the survivor set $\bar{\Delta}^{\infty}$. Recall from $\S 5.1$ that modulo a set of $\bar{m}$-measure zero, $\bar{\Delta}_{0}$ is the disjoint union of a countable number of closed subsets $\left(\bar{\Delta}_{0}\right)_{j}$ with the properties that:

(i) in the absence of $\bar{H}, \bar{F}^{R}$ maps each $\left(\bar{\Delta}_{0}\right)_{j}$ homeomorphically onto $\bar{\Delta}_{0}$; and

(ii) with $\bar{H}$ present, each $\left(\bar{\Delta}_{0}\right)_{j}$ either falls entirely into $\bar{H}$ on its way up the tower or returns to $\bar{\Delta}_{0}$ intact.

We rename the subcollection $\left\{\left(\bar{\Delta}_{0}\right)_{j}\right\}$ that returns to $\bar{\Delta}_{0}$ in (ii) as $\left\{A_{i}\right\}$, and define

$$
\bar{\Delta}_{0}^{\infty}:=\bar{\Delta}^{\infty} \cap \bar{\Delta}_{0}=\bigcap_{n \geq 0}\left(\bar{F}^{R}\right)^{-n}\left(\bigcup_{i} A_{i}\right) .
$$

It is easy to see that there is a bijection $\pi_{0}: \bar{\Delta}_{0}^{\infty} \rightarrow \Sigma_{\infty}=\Pi_{i=1}^{\infty}\{1,2,3, \ldots\}$ such that $\pi_{0} \circ \bar{F}^{R}=T \circ \pi_{0}$, where $T: \Sigma_{\infty} \circlearrowleft$ is the full shift. Moreover, with $\bar{\Delta}_{0}^{\infty}$, given its relative topology as a subset of $\bar{\Delta}_{0}$, and $\Sigma_{\infty}$, given the topology defined by cylinder sets, $\pi_{0}$ is a conjugating homeomorphism.

Let $\mathcal{Z}_{n}$ denote the set of cylinders in $\Sigma_{\infty}$ defined by coordinates $1, \ldots, n$, and write $\mathcal{Z}=\mathcal{Z}_{1}$. We introduce a metric $\hat{d}$ on $\Sigma_{\infty}$ compatible with its topology, defined by $\left\{\mathcal{Z}_{n}\right\}:$ for $x, y \in \Sigma_{\infty}$, define $\hat{s}(x, y)=\min \left\{i \in \mathbb{N} \mid T^{i} x, T^{i} y\right.$ lie in different $\left.Z \in \mathcal{Z}\right\}$, and let $\hat{d}(x, y)=\beta^{\hat{s}(x, y)}$ (where $\beta$ is as in $\S 5.1$ ). We say a function $\phi: \Sigma_{\infty} \rightarrow \mathbb{R}$ is locally Hölder continuous if

$$
\sup _{Z \in \mathcal{Z}}\left\{|\phi(x)-\phi(y)| \cdot \beta^{-\hat{s}(x, y)}: x, y \in Z\right\}<\infty .
$$


2. Sarig's abstract results on the pressure of $T: \Sigma_{\infty} \circlearrowleft$. We recall here a few relevant results for $T: \Sigma_{\infty} \circlearrowleft$. These results were proved in $[\mathbf{S}]$ in more general settings of topologically mixing countable Markov shifts. Given $\phi: \Sigma_{\infty} \rightarrow \mathbb{R}$, let $S_{n} \phi=\sum_{i=0}^{n-1} \phi$ 。 $T^{i}$. The Gurevic pressure of $\phi$ is defined to be

$$
P_{G}(\phi, Z)=\lim _{n \rightarrow \infty} \frac{1}{n} \log \left(\sum_{T^{n} x=x ; x \in Z} e^{S_{n} \phi(x)}\right),
$$

where $Z$ is any fixed element of $\mathcal{Z}$. For $\phi$ locally Hölder continuous, it is shown in [S, Theorem 1] that the limit above exists and is independent of $Z$. This number is $\leq \infty$ in general, and is equal to $\infty$ for many $\phi$, given that $T$ is an infinite shift.

We will also need the following definitions: the transfer operator associated with $\phi$ is given by

$$
\mathcal{L}_{\phi} \psi(x)=\sum_{T y=x} e^{\phi(y)} \psi(y) \quad \text { for bounded } \psi .
$$

Let $\mathcal{M}_{T}\left(\Sigma_{\infty}\right)$ be the set of $T$-invariant Borel probability measures on $\Sigma_{\infty}$. Given a potential $\phi: \Sigma_{\infty} \rightarrow \mathbb{R}$, we say $\eta \in \mathcal{M}_{T}\left(\Sigma_{\infty}\right)$ is a Gibbs measure for $\phi$ if there exist constants $C>1$ and $P_{\eta} \in \mathbb{R}$ such that for any $n \geq 1, Z_{n} \in \mathcal{Z}_{n}$ and $x \in Z_{n}$,

$$
C^{-1} e^{S_{n} \phi(x)-n P_{\eta}} \leq \eta\left(Z_{n}\right) \leq C e^{S_{n} \phi(x)-n P_{\eta}} .
$$

The following version of results from $[\mathbf{S}]$ are adequate for our purposes.

TheOREM 6. Let $T: \Sigma_{\infty} \circlearrowleft$ be as above, and let $\phi: \Sigma_{\infty} \rightarrow \mathbb{R}$ be locally Hölder continuous. Assume $\left|\mathcal{L}_{\phi} 1\right|_{\infty}<\infty$. Then:

(a) $\left[\mathbf{S}\right.$, Theorem 1] $P_{G}(\phi)<\infty$;

(b) $[\mathbf{S}$, Theorem 3]

$$
P_{G}(\phi)=\sup \left\{h_{\eta}(T)+\int \phi d \eta \mid \eta \in \mathcal{M}_{T}\left(\Sigma_{\infty}\right) \text { and } \eta(-\phi)<\infty\right\} ;
$$

(c) [S, Theorem 8] Suppose $\eta$ is a Gibbs measure for $\phi$, and $\eta(-\phi)<\infty$; then

$$
P_{G}(\phi)=P_{\eta}=h_{\eta}(T)+\int \phi d \eta
$$

It follows from (a) and (b) above that for $\eta \in \mathcal{M}_{T}\left(\Sigma_{\infty}\right), h_{\eta}(T)<\infty$, provided $\left|\mathcal{L}_{\phi} 1\right|_{\infty}<\infty$ and $\eta(-\phi)<\infty$.

Notation. In what follows, we will identify $\bar{F}^{R}: \bar{\Delta}_{0}^{\infty} \circlearrowleft$ with $T: \Sigma_{\infty} \circlearrowleft$ and use the two sets of notations interchangeably. We also introduce the following notation: given $\bar{\eta} \in \mathcal{M}_{\bar{F}}\left(\bar{\Delta}^{\infty}\right)$, let $\bar{\eta}_{0}$ denote the measure $\left.\left(\left(1 / \bar{\eta}\left(\bar{\Delta}_{0}^{\infty}\right)\right) \bar{\eta}\right)\right|_{\bar{\Delta}_{0}^{\infty}}$. It is easy to see that $\bar{\eta}_{0} \in \mathcal{M}_{\bar{F}^{R}}\left(\bar{\Delta}_{0}^{\infty}\right)$.

3. Relating pressure on $\left(\bar{F}, \bar{\Delta}^{\infty}\right)$ to that on $\left(\bar{F}^{R}, \bar{\Delta}_{0}^{\infty}\right)$. Let $\phi=-\log \left(\mathfrak{r}^{R} J \bar{F}^{R}\right)$. The aim of this step is to prove that for every $\bar{\eta} \in \mathcal{M}_{\bar{F}}\left(\bar{\Delta}^{\infty}\right)$ with $\bar{\eta}(\log J \bar{F})<\infty$,

$$
\bar{\eta}\left(\bar{\Delta}_{0}^{\infty}\right)^{-1}\left\{h_{\bar{\eta}}(\bar{F})-\bar{\eta}(\log J \bar{F})-\log \mathfrak{r}\right\}=h_{\bar{\eta}_{0}}\left(\bar{F}^{R}\right)+\bar{\eta}_{0}(\phi) \leq P_{G}(\phi)<\infty .
$$

The last two inequalities follow from Theorem 6(a) and (b) once we check (i) $\phi$ is locally Hölder continuous with respect to the metric $\hat{d}$, (ii) $\left|\mathcal{L}_{\phi} 1\right|_{\infty}<\infty$, and (iii) $\bar{\eta}_{0}(-\phi)<\infty$. 
For (i), notice that by Lemma 5.1, $\phi$ is locally Hölder continuous with respect to the separation time metric $d_{\beta}$, and $\hat{s}(x, y) \leq s(x, y)$.

For (ii), let $Z(y)$ denote the element of $\mathcal{Z}$ containing $y \in \bar{\Delta}_{0}^{\infty}$. We fix $x \in \bar{\Delta}_{0}^{\infty}$ and use the bounded distortion of $J \bar{F}^{R}$ given by Lemma 5.1(c) to write

$$
\begin{aligned}
\mathcal{L}_{\phi} 1(x) & =\sum_{T y=x} \mathfrak{r}^{-R(y)}\left(J \bar{F}^{R}(y)\right)^{-1} \leq C \sum_{T y=x} \mathfrak{r}^{-R(y)} \bar{m}(Z(y)) \\
& \leq C \sum_{n \geq 1} \mathfrak{r}^{-n} \bar{m}\{R=n\} \leq C^{\prime} \sum_{n \geq 1} \mathfrak{r}^{-n} \theta_{0}^{n}<\infty .
\end{aligned}
$$

Here, we have used $\mathfrak{r}>\theta_{0}$ and the fact that $\bar{F}^{R}$ maps each $Z \in \mathcal{Z}$ bijectively onto $\bar{\Delta}_{0}^{\infty}$.

For (iii), we will show $\bar{\eta}(\log J \bar{F})<\infty$ implies $\bar{\eta}_{0}(-\phi)<\infty$ : since $J \bar{F} \equiv 1$ on $\bar{\Delta} \backslash \bar{F}^{-1} \bar{\Delta}_{0}$,

$$
\int_{\bar{\Delta}_{0}^{\infty}} \log J \bar{F}^{R} d \bar{\eta}_{0}=\bar{\eta}\left(\bar{\Delta}_{0}^{\infty}\right)^{-1} \int_{\bar{F}^{-1} \bar{\Delta}_{0}^{\infty}} \log J \bar{F} d \bar{\eta}=\bar{\eta}\left(\bar{\Delta}_{0}^{\infty}\right)^{-1} \int_{\bar{\Delta}^{\infty}} \log J \bar{F} d \bar{\eta}
$$

Thus, if $\bar{\eta}(\log J \bar{F})<\infty$, then, noting $\bar{\eta}\left(\bar{\Delta}_{0}^{\infty}\right) \int R d \bar{\eta}_{0}=1$, we have

$$
\begin{aligned}
\bar{\eta}_{0}(-\phi) & =\int_{\bar{\Delta}_{0}^{\infty}} \log \left(\mathfrak{r}^{R} J \bar{F}^{R}\right) d \bar{\eta}_{0}=\int_{\bar{\Delta}_{0}^{\infty}} R \log \mathfrak{r} d \bar{\eta}_{0}+\int_{\bar{\Delta}_{0}^{\infty}} \log J \bar{F}^{R} d \bar{\eta}_{0} \\
& =(\log \mathfrak{r}+\bar{\eta}(\log J \bar{F})) \cdot \bar{\eta}\left(\bar{\Delta}_{0}^{\infty}\right)^{-1}<\infty .
\end{aligned}
$$

This completes the verification of (i)-(iii).

The equality in (12) follows from (13) together with the general formula of Abramov [A], which says that $h_{\bar{\eta}}(\bar{F})=h_{\bar{\eta}_{0}}\left(\bar{F}^{R}\right) \bar{\eta}\left(\bar{\Delta}_{0}^{\infty}\right)$. In all the references we know of (e.g. [Pe, §6.1]), this equality is proved assuming the invertibility of the transformation. In the situation above, $\bar{F}$ is clearly not invertible, but the same result is easily deduced by passing to natural extensions; see Appendix B.

4. Existence of a pressure-maximizing invariant measure $\bar{v}$. Let $\bar{v}$ be the linear functional on $C_{b}^{0}(\bar{\Delta})$ defined by

$$
\bar{v}(\psi)=\lim _{n \rightarrow \infty} \mathfrak{r}^{-n} \int_{\bar{\Delta}} \overline{\mathcal{L}}^{n}\left(h_{*} \psi\right) d \bar{m}=\lim _{n \rightarrow \infty} \mathfrak{r}^{-n} \int_{\bar{\Delta}^{n}} \psi h_{*} d \bar{m} .
$$

We refer the reader to $[\mathbf{B D M}]$ for verification that $\bar{v}$ is a well defined, $\bar{F}$-invariant probability measure on $\bar{\Delta}^{\infty}$.

The aim of this step is to show that plugging $\bar{\eta}=\bar{v}$ into (12), we get

$$
h_{\bar{\nu}_{0}}\left(\bar{F}^{R}\right)+\bar{v}_{0}(\phi)=P_{G}(\phi)=0 \quad \text { and } \quad h_{\bar{\nu}}(\bar{F})-\bar{v}(\log J \bar{F})=\log \mathfrak{r} .
$$

Observe from the definition of $\phi$ in step 3 that $e^{S_{n} \phi(x)}=\mathfrak{r}^{-S_{n} R(x)}\left(J\left(\bar{F}^{R}\right)^{n}(x)\right)^{-1}$. The following lemma shows that $\bar{\nu}_{0}$ is a Gibbs measure for the potential $\phi$, with $P_{\bar{\nu}_{0}}=0$.

LEMMA 5.2. [BDM, Lemma 5.3] There exists a constant $C>1$ such that for any $n \geq 1$, any $n$-cylinder $Z_{n} \in \mathcal{Z}_{n}$, and any $y_{*} \in Z_{n}$,

$$
C^{-1} \mathfrak{r}^{-S_{n} R\left(y_{*}\right)}\left(J\left(\bar{F}^{R}\right)^{n}\left(y_{*}\right)\right)^{-1} \leq \bar{\nu}_{0}\left(Z_{n}\right) \leq C \mathfrak{r}^{-S_{n} R_{n}\left(y_{*}\right)}\left(J\left(\bar{F}^{R}\right)^{n}\left(y_{*}\right)\right)^{-1} .
$$


It remains only to check that $\bar{v}(\log J \bar{F})<\infty$, for this bound implies $\bar{v}_{0}(-\phi)<\infty$ (see step 3 above), and once we have that, Theorem 6(c) gives the first equation in (14). The second equation follows from (12) and the first.

In what follows, $C$ will be used as a generic constant, the value of which is permitted to vary from line to line. To prove $\bar{v}(\log J \bar{F})<\infty$, we first estimate

$$
\begin{aligned}
\bar{v}_{0}\{R=n\} & =\sum_{Z \in \mathcal{Z}: R(Z)=n} \bar{v}_{0}(Z) \leq \sum_{Z \in \mathcal{Z}: R(Z)=n} C \mathfrak{r}^{-n}\left(J \bar{F}^{R}\left(y_{*}\right)\right)^{-1} \\
& \leq C \sum_{Z \in \mathcal{Z}: R(Z)=n} \mathfrak{r}^{-n} \bar{m}(Z) \leq C \theta_{0}^{n} \mathfrak{r}^{-n}
\end{aligned}
$$

where $y_{*}$ is an arbitrary point in $Z$. The first inequality comes from Lemma 5.2, the second from Lemma 5.1(c), and the third from the tail bound for $(\bar{\Delta}, \bar{F})$. Using the invariance of $\bar{v}$ and the fact that $J \bar{F} \equiv 1$ on $\bar{\Delta} \backslash \bar{F}^{-1}(\bar{\Delta})$, we obtain

$$
\begin{aligned}
\bar{v}(\log (J \bar{F})) & =\sum_{n \geq 1} \sum_{R(Z)=n} \int_{Z} \log \left(J \bar{F}^{n}\right) d \bar{v}=\sum_{n \geq 1} \bar{v}\{R=n\}\left|\log J \bar{F}^{n}\right|_{\infty} \\
& \leq C \sum_{n \geq 1}\left(\theta_{0} \mathfrak{r}^{-1}\right)^{n} \bar{\theta}^{-n}<\infty .
\end{aligned}
$$

The inequalities above come from condition $(*)$ in Theorem 3 ; this is the only place in the entire proof that uses this condition. We have also used the fact that $\bar{\nu}\{R=n\}$ is bounded by $\bar{v}\left(\bar{\Delta}_{0}^{\infty}\right)$ times the last quantity in (15).

Parts (b) and (c) of Theorem 3 follow immediately from steps 3 and 4.

5.4. Proof of Theorem 5. We will prove this theorem by leveraging the corresponding results for expanding towers.

Variational principle (Theorem 5(a), (b)). First, we show

$$
\sup _{\eta \in \mathcal{G}_{\Delta}}\left\{h_{\eta}(F)-\int \log J_{\mu}^{u}(F) d \eta\right\} \leq \sup _{\bar{\eta} \in \mathcal{G}_{\bar{\Delta}}}\left\{h_{\bar{\eta}}(\bar{F})-\int \log J(\bar{F}) d \bar{\eta}\right\},
$$

which follows immediately from the following lemma.

Lemma 5.3. Let $\eta \in \mathcal{G}_{\Delta}$ and define $\bar{\eta}=\bar{\pi}_{*} \eta$. Then $\bar{\eta} \in \mathcal{G}_{\bar{\Delta}}$ and:

(i) $\int_{\Delta} \log J_{\mu}^{u} F d \eta=\int_{\bar{\Delta}} \log J \bar{F} d \bar{\eta}$;

(ii) $h_{\eta}(F)=h_{\bar{\eta}}(\bar{F})$.

Proof of Lemma 5.3. Let $\eta \in \mathcal{G}_{\Delta}$. The fact that $\bar{\eta}=\bar{\pi}_{*} \eta \in \mathcal{M}_{\bar{F}}\left(\bar{\Delta}^{\infty}\right)$ is clear. That $\bar{\eta} \in \mathcal{G}_{\bar{\Delta}}$ will follow once we prove assertion (i) of the lemma: from §5.1.I, we see that $\log J_{\mu}^{u} F$ and $J \bar{F}$ are related by $J \bar{F} \circ \bar{\pi}=J_{\mu}^{u} F \cdot e^{a \circ F-a}$ for a bounded function $a$ (Lemma 5.1(d)). It follows that

$$
\int_{\Delta} \log J_{\mu}^{u} F d \eta=\int_{\Delta}(\log J \bar{F} \circ \bar{\pi}+a-a \circ F) d \eta=\int_{\bar{\Delta}} \log J \bar{F} d \bar{\eta},
$$

the invariance of $\eta$ being used in the second equality.

Assertion (ii) follows from (a) the entropy of a transformation is equal to that of its natural extension, and (b) the natural extension of $(F, \eta)$ is isomorphic to that of $(\bar{F}, \bar{\eta})$. See Appendix B for more detail on (b). 
To complete the proof, we will show that (i) the results of Theorem 3 are applicable to the quotient tower, and (ii) $\tilde{v}$ as defined in part (b) is in $\mathcal{G}_{\Delta}$ and projects to $\bar{v}$. These two steps together will show that (16) is in fact an equality, and the quantity on the right is $=\log \mathfrak{r}$.

To apply Theorem 3, it suffices to show that condition $(*)$ holds in the present setting, i.e. for the quotient tower of a hyperbolic tower arising from $(f, M ; H)$ and satisfying (A.1)-(A.4). Notice first that (*) holds if $\|D f\|$ is bounded, for $J_{\mu}^{u} F^{n}$ can grow at most exponentially, and $J \bar{F}^{n}$ on the corresponding set is $\leq J_{\mu}^{u} F^{n} \cdot e^{|a|_{\infty}}$, where $a$ is as in Lemma 5.3. Thus, there is a potential problem only in the setting of Theorem $\mathrm{C}$, where $\|D f\|$ may become arbitrarily large as one approaches the singularity set $\mathcal{S}$. Here, it is (H.2) of §5.1.II and (3) in §2.1.III that give what we need: since $F^{-j} \Delta_{j}=\{x \in \Lambda: R(x)>$ $j\}$, we have $d\left(\pi \Delta_{j}, \mathcal{S}\right) \geq \delta \xi_{1}^{-j}$ for some $\delta>0$ and $\xi_{1}>1$ by (H.2). This, together with (3), implies that on $\pi\left(\Delta_{j}\right),\left|\operatorname{det}\left(\left.D f\right|_{E^{u}}\right)\right| \leq\left(C_{1} \delta^{-a} \xi_{1}^{a j}\right)^{p}$, where $p$ is the dimension of $E^{u}$. Thus, on $\Delta_{0} \cap\{R=n\}$, we have

$$
\log J_{\mu}^{u} F^{n}=\sum_{j=0}^{n-1} \log \left|\operatorname{det}\left(\left.D f\right|_{E^{u}}\right) \circ f^{j}\right| \leq \text { const } n^{2},
$$

which, as explained above, gives $(*)$.

It remains to produce $\tilde{v}$ with the properties in (ii). Let $\tilde{\mu}_{*}$ be the physical conditionally invariant distribution from Theorem 4. For $\varphi \in \operatorname{Lip}^{u}(\Delta)$, define $\tilde{\mu}^{\varphi}$ to be the measure such that $d \tilde{\mu}^{\varphi}=\varphi d \tilde{\mu}_{*}$. Notice that since $\bar{\pi}_{*} \tilde{\mu}_{*} \in \mathcal{B}$ and $|\varphi|_{\infty}+|\varphi|_{\text {Lip }}^{u}<\infty$, we also have $\bar{\pi}_{*} \tilde{\mu}^{\varphi} \in \mathcal{B}$. Let $\bar{\psi}_{\varphi}$ denote the density of $\bar{\pi}_{*} \tilde{\mu}^{\varphi}$ with respect to $\bar{m}$. Now, using Theorem 4(b),

$$
\lim _{n \rightarrow \infty} \mathfrak{r}^{-n} \int_{\Delta^{n}} \varphi d \tilde{\mu}_{*}=\lim _{n \rightarrow \infty} \mathfrak{r}^{-n} \tilde{\mu}^{\varphi}\left(1_{\Delta^{n}}\right)=\lim _{n \rightarrow \infty} \mathfrak{r}^{-n} \stackrel{\circ}{F}_{*}^{n} \tilde{\mu}^{\varphi}(1)=d\left(\bar{\psi}_{\varphi}\right) .
$$

Let $Q(\varphi)=d\left(\bar{\psi}_{\varphi}\right)$. Then $Q$ is clearly linear in $\varphi$, positive and satisfies $Q(1)=1$. Also, $|Q(\varphi)| \leq|\varphi|_{\infty} Q(1)$ so that $Q$ extends to a bounded linear functional on $C_{b}^{0}(\Delta)$. By the Riesz representation theorem, there exists a unique Borel probability measure $\tilde{v}$ satisfying $\tilde{v}(\varphi)=Q(\varphi)$ for each $\varphi \in C_{b}^{0}(\Delta)$. Since $1_{\Delta^{n}}=1_{\Delta^{n-1}} \circ \stackrel{\circ}{F}$, the invariance of $\tilde{v}$ follows from

$$
\begin{aligned}
\tilde{v}(\varphi \circ \stackrel{\circ}{F}) & =\lim _{n \rightarrow \infty} \mathfrak{r}^{-n} \tilde{\mu}_{*}\left(\varphi \circ \stackrel{\circ}{F} \cdot 1_{\Delta^{n}}\right)=\lim _{n \rightarrow \infty} \mathfrak{r}^{-n} \stackrel{\circ}{F}_{*} \tilde{\mu}_{*}\left(\varphi \cdot 1_{\Delta^{n-1}}\right) \\
& =\lim _{n \rightarrow \infty} \mathfrak{r}^{1-n} \tilde{\mu}_{*}\left(\varphi \cdot 1_{\Delta^{n-1}}\right)=\tilde{v}(\varphi),
\end{aligned}
$$

by the conditional invariance of $\tilde{\mu}_{*}$.

Since $\left.\left(\bar{\pi}_{*} \tilde{\mu}_{*}\right)\right|_{\Delta^{n}}=\left.\left(h_{*} \bar{m}\right)\right|_{\bar{\Delta}^{n}}$ for every $n$, it follows that $\bar{\pi}_{*} \tilde{v}=\bar{v}$. To place $\tilde{v} \in \mathcal{G}_{\Delta}$, we need to show $\tilde{v}\left(\log J_{\mu}^{u} F\right)<\infty$. This is true by (17) with $\eta=\tilde{v}$ and the fact that the integral on the right is known to be finite.

Other properties of $\tilde{v}$. The ergodicity of $\tilde{v}$ follows from that of $\bar{v}$. To show that $\tilde{v}$ enjoys exponential decay of correlations, we begin by decomposing $\tilde{v}$ into conditional measures $\tilde{v}^{s}$ on $\omega^{s}$-leaves and a transverse measure $\tilde{v}_{T}$ on the set of stable leaves in each $\Delta_{\ell, j}$. For $\varphi \in C_{b}^{0}$, define $\bar{\varphi}(x)=\int_{\omega^{s}(x)} \varphi d \tilde{\nu}^{s}$. Since each $\tilde{\nu}^{s}$ is a probability measure, we 
have $\bar{\varphi} \in C_{b}^{0}$. By definition, $\bar{\varphi}$ is constant on $\omega^{s}$-leaves and $\tilde{v}(\varphi)=\tilde{v}(\bar{\varphi})=\bar{v}(\bar{\varphi})$. Also, if $\varphi \in \operatorname{Lip}^{u}(\Delta)$, then $\bar{\varphi} \in \operatorname{Lip}^{u}(\Delta)$ so that we may consider $\bar{\varphi} \in \mathcal{B}_{0}$ as a function on $\bar{\Delta}$.

Now let $\varphi \in \operatorname{Lip}^{u}(\Delta)$ and $\psi \in \operatorname{Lip}^{s}(\Delta)$ with $\tilde{v}(\varphi)=\tilde{v}(\psi)=0$. Define $\bar{\varphi}$ as above and let $\bar{\psi}_{k}(x)=\int_{\omega^{s}(x)} \psi \circ F^{k} d \tilde{v}^{s}$. Note that $\tilde{v}\left(\bar{\psi}_{k}\right)=\tilde{v}(\psi)=0$. Then, setting $n=k+\ell$, we write

$$
\tilde{v}\left(\varphi \psi \circ F^{n}\right)=\tilde{v}\left(\varphi\left(\psi \circ F^{n}-\bar{\psi}_{k} \circ F^{\ell}\right)\right)+\tilde{v}\left((\varphi-\bar{\varphi}) \bar{\psi}_{k} \circ F^{\ell}\right)+\tilde{v}\left(\bar{\varphi}_{k} \circ F^{\ell}\right) .
$$

Since $\bar{\varphi}$ and $\bar{\psi}_{k}$ are constant on $\omega^{s}$-leaves, we have $\tilde{v}\left(\bar{\varphi}_{\bar{\psi}} \circ \bar{\psi}^{\ell}\right)=\bar{\nu}\left(\bar{\varphi}_{\bar{\psi}} \circ \bar{F}^{\ell}\right)$ and $\bar{v}(\bar{\varphi})=\bar{v}\left(\bar{\psi}_{k}\right)=0$. Then, since $\bar{\varphi} \in \mathcal{B}_{0}$ and $\bar{\psi}_{k} \in L^{\infty}(\bar{\Delta})$, the last term in (18) is $\leq C \tau^{\ell}\|\bar{\varphi}\|_{\mathcal{B}_{0}}|\bar{\psi}|_{\infty}$ for some $\tau<1$ by Theorem 3(d) (see also [BDM, Proposition 2.8]).

The second term of (18) is identically 0 since,

$$
\begin{aligned}
\tilde{v}\left(\varphi \bar{\psi}_{k} \circ F^{\ell}\right) & =\int_{\Gamma^{s}(\Delta)}\left(\int_{\omega^{s}} \varphi \bar{\psi}_{k} \circ F^{\ell} d \tilde{\nu}^{s}\right) d \tilde{v}_{T}=\int_{\Gamma^{s}(\Delta)}\left(\int_{\omega^{s}} \varphi d \tilde{v}^{s}\right) \bar{\psi}_{k} \circ F^{\ell} d \tilde{v}_{T} \\
& =\int_{\Gamma^{s}(\Delta)} \bar{\varphi} \bar{\psi}_{k} \circ F^{\ell} d \tilde{v}_{T}=\tilde{v}\left(\bar{\varphi} \bar{\psi}_{k} \circ F^{\ell}\right) .
\end{aligned}
$$

To estimate the first term in (18), notice that $\left|\psi \circ F^{n}-\bar{\psi}_{k} \circ F^{\ell}\right|_{\infty} \leq\left|\psi \circ F^{k}-\bar{\psi}_{k}\right|_{\infty}$. Then, since $\psi \circ F^{k}$ is continuous on each $\omega^{s}$, there must exist $x, y \in \omega^{s}$ such that $\psi \circ F^{k}(x) \leq \bar{\psi}_{k}\left(\omega^{s}\right) \leq \psi \circ F^{k}(y)$. Thus,

$$
\left|\tilde{v}\left(\varphi\left(\psi \circ F^{n}-\bar{\psi}_{k} \circ F^{\ell}\right)\right)\right| \leq|\varphi|_{\infty}\left|\psi \circ F^{k}-\bar{\psi}_{k}\right|_{\infty} \leq 2|\varphi|_{\infty}|\psi|_{\text {Lip }}^{s} C \alpha^{k},
$$

by definition of $d_{s}$. Taking both $k$ and $\ell$ to be approximately $n / 2$ completes the proof.

\section{Proof of Theorems $D$ and $E$}

We now return to the original open system $(f, M ; H)$, where $f$ is any dynamical system admitting a tower with the properties in $\$ 2.2$ (see $\$ 5.1$ for details).

6.1. Proof of Theorem $D$. Let $\tilde{\mu}_{\mathrm{SRB}}$ be the SRB measure for $F$ on $\Delta$ before the removal of the hole. Note that $\pi_{*} \tilde{\mu}_{\mathrm{SRB}}=\mu_{\mathrm{SRB}}$, the unique SRB measure for $f$ with $\mu_{\mathrm{SRB}}(\Lambda)>0$. It follows from $[\mathbf{Y 3}, \S 2]$ that $\tilde{\mu}_{\mathrm{SRB}} \in \tilde{\mathcal{B}}$, so that $\rho\left(\tilde{\mu}_{\mathrm{SRB}}\right)=\log \mathfrak{r}$ by Theorem 4(a). Since $\mu_{\mathrm{SRB}}\left(M^{n}\right)=\pi_{*} \tilde{\mu}_{\mathrm{SRB}}\left(M^{n}\right)=\tilde{\mu}_{\mathrm{SRB}}\left(\Delta^{n}\right)$ for each $n \geq 0$, we have $\rho\left(\mu_{\mathrm{SRB}}\right)=\log \mathfrak{r}$ and part (a) of Theorem $\mathrm{D}$ is proved.

To prove part (b), define $\mu_{*}=\pi_{*} \tilde{\mu}_{*}$, where $\tilde{\mu}_{*}$ is the conditionally invariant measure from Theorem 4. We use $f^{n}=\left.f^{n}\right|_{M^{n}}$ to describe the surviving dynamics at time $n$. It follows from the relation $f \circ \pi=\pi \circ \stackrel{\circ}{f}$ that for any Borel subset $A$ of $M \backslash H$, we have

$$
\mu_{*}\left(\stackrel{\circ}{f}^{-1} A\right)=\tilde{\mu}_{*}\left(\pi^{-1}\left(\stackrel{\circ}{f}^{-1} A\right)\right)=\tilde{\mu}_{*}\left(\stackrel{\circ}{F}^{-1}\left(\pi^{-1} A\right)\right)=\mathfrak{r} \tilde{\mu}_{*}\left(\pi^{-1} A\right)=\mathfrak{r} \mu_{*}(A),
$$

so that $\mu_{*}$ is a conditionally invariant measure for $f$ with eigenvalue $\mathfrak{r}$. By Theorem $4(\mathrm{~b})$,

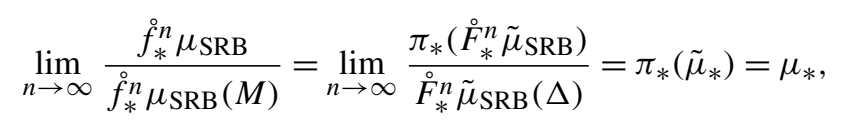

proving part (b).

To prove part (c), define $\hat{v}=\pi_{*} \tilde{\nu}$, where $\tilde{v}$ is from Theorem 5. Arguing analogously to (20), we see that $\hat{v}$ is an invariant measure for $f$ supported on $\pi\left(\Delta^{\infty}\right) \subseteq \Omega$. Write $J^{u} f(x)=\left|\operatorname{det}\left(\left.D f_{x}\right|_{E^{u}(x)}\right)\right|$. We will show: 
(i) $\int_{\Delta} \log J_{\mu}^{u} F d \tilde{v}=\int_{M} \log J^{u} f d \hat{v}$; and

(ii) $h_{\tilde{v}}(F)=h_{\hat{v}}(f)$.

Integrating over sets of the form $\bigcup_{i=0}^{n-1} F^{i}\left(\Delta_{0} \cap\{R=n\}\right)$ before summing over $n$, we see that the left side of (i) is equal to $\int_{\Delta_{0}} \log J_{\mu}^{u} F^{R} d \tilde{v}$ and the right side is equal to $\int_{M} \log J^{u} f^{R} d \pi_{*}\left(\left.\tilde{v}\right|_{\Delta_{0}}\right)$, the latter using the invariance of $\tilde{v}$ and the relation $\pi_{*}\left(F^{i}\right)_{*}=$ $\left(f^{i}\right)_{*} \pi_{*}$. These two integrals are easily seen to be equal: let $J^{u} \pi$ denote the Jacobian with respect to $\tilde{\mu}_{\omega}$ for $\omega \in \Gamma^{u}(\Delta)$ and $\mu_{\omega^{\prime}}$, where $\pi(\omega)=\omega^{\prime}$. Then, on $\Delta_{0}, J^{u} \pi \equiv 1$, as $\Delta_{0}$ is an isometric copy of $\Lambda$, we have

$$
J^{u} f^{R} \circ \pi=J_{\mu}^{u} F^{R} \cdot \frac{J^{u} \pi \circ F^{R}}{J^{u} \pi}=J_{\mu}^{u} F^{R} .
$$

For (ii), that $h_{\hat{v}}(f) \leq h_{\tilde{v}}(F)$ is obvious. The reverse inequality follows from [Bu, Proposition 2.8] since $\pi$ is at most countable-to-one. Combining (i) and (ii) and using Theorem 5(b),

$$
\rho\left(\mu_{\mathrm{SRB}}\right)=\log \mathfrak{r}=h_{\tilde{v}}(F)-\int_{\Delta} \log J_{\mu}^{u} F d \tilde{v}=h_{\hat{v}}(f)-\int_{M} \log J^{u} f d \hat{v}=P_{\hat{v}} .
$$

The following lemma completes the proof of part (c).

LEMMA 6.1. $\hat{v} \in \mathcal{G}_{H} \cap \mathcal{G}_{\mathcal{S}}$.

Proof. That $\hat{v}$ is ergodic follows immediately from the fact that $\tilde{v}$ is ergodic. In order to show that $\hat{v} \in \mathcal{G}_{H} \cap \mathcal{G}_{\mathcal{S}}$, we will show that there exist $C, \alpha>0$ such that for each $\varepsilon>0$, $\hat{v}\left(N_{\varepsilon}(\mathcal{S} \cup \partial H)\right) \leq C \varepsilon^{\alpha}$. Once this is established, we conclude by an argument similar to Lemma 4.2 that $\hat{v}$-a.e. point approaches $\mathcal{S} \cup \partial H$ at an arbitrarily slow exponential rate.

To establish this bound, we need estimates on how $\tilde{v}$ decays up the levels of the tower. Recall $\bar{v}=\bar{\pi}_{*} \tilde{\nu}$. In the proof of Theorem 5, we established that $d\left(\pi \Delta_{\ell}, \mathcal{S} \cup \partial H\right) \geq \delta \xi_{1}^{-\ell}$, $\ell \geq 0$, by using (H.2) of §5.1.II. Thus, we have

$$
\hat{v}\left(N_{\varepsilon}(\mathcal{S} \cup \partial H)\right) \leq \tilde{v}\left(\bigcup_{\ell: \delta \xi_{1}^{-\ell} \leq \varepsilon} \Delta_{\ell}\right) \leq \sum_{\ell \geq \log (\delta / \varepsilon) / \log \xi_{1}} C^{\prime} \theta_{0}^{\ell} \mathfrak{r}^{-\ell} \leq C^{\prime \prime}\left(\delta^{-1} \varepsilon\right)^{\log \left(r_{0}^{-1}\right) / \log \xi_{1}},
$$

using (15) and $\tilde{v}\left(\Delta_{\ell}\right)=\bar{v}\left(\bar{\Delta}_{\ell}\right)$.

Finally, we prove part (d). If $\varphi$ is a continuous function on $M$, we define its lift to $\Delta$ by $\tilde{\varphi}=\varphi \circ \pi$. This lift is continuous on each $\Delta_{\ell, j}$ and $|\tilde{\varphi}|_{\infty} \leq|\varphi|_{\infty}$, so that $\tilde{\varphi} \in C_{b}^{0}(\Delta)$. Using Theorem 5(b), we have

$$
\hat{v}(\varphi)=\tilde{v}(\tilde{\varphi})=\lim _{n \rightarrow \infty} \mathfrak{r}^{-n} \int_{\Delta^{n}} \tilde{\varphi} d \tilde{\mu}_{*}=\lim _{n \rightarrow \infty} \mathfrak{r}^{-n} \int_{M^{n}} \varphi d \mu_{*},
$$

since $\mu_{*}=\pi_{*} \tilde{\mu}_{*}$.

To complete the proof of Theorem $\mathrm{D}$, it remains to show that $\hat{v}$ enjoys exponential decay of correlations. Let $C^{p}(M)$ denote the Hölder continuous functions on $M$ with exponent $p$. If $\varphi \in C^{p}(M)$ and $p \geq \log \beta / \log \alpha$, then $\varphi \circ \pi \in \operatorname{Lip}^{u}(\Delta)$. This can be proved as in [D, §6]. Also, taking $\psi \in C^{p}(M)$, for $x \in \Delta_{\ell, j}, y \in \omega^{s}(x)$ and $x_{0}=F^{-\ell} x, y_{0}=F^{-\ell} y$, we have

$$
\begin{aligned}
\left|\psi \circ \pi \circ F^{n}(x)-\psi \circ \pi \circ F^{n}(y)\right| & \leq|\psi|_{C^{p}} d\left(\pi\left(F^{n+\ell} x_{0}\right), \pi\left(F^{n+\ell} y_{0}\right)\right)^{p} \\
& \leq|\psi|_{C^{p}} d\left(f^{n+\ell}\left(\pi x_{0}\right), f^{n+\ell}\left(\pi y_{0}\right)\right)^{p} \leq|\psi|_{C p} C \alpha^{n p} .
\end{aligned}
$$


So, taking $\varphi, \psi \in C^{p}(M)$, we may apply (19) to $\psi \circ \pi$. We follow (18), and note that

$$
\hat{v}\left(\varphi \psi \circ f^{n}\right)=\tilde{v}\left(\varphi \circ \pi \cdot \psi \circ f^{n} \circ \pi\right)=\tilde{v}\left(\varphi \circ \pi \cdot \psi \circ \pi \circ F^{n}\right),
$$

to conclude that the exponential decay of correlations for $\hat{v}$ follows from that for $\tilde{v}$.

6.2. Proof of Theorem E. As an immediate corollary of Theorem D, we have

$$
\rho\left(\mu_{\mathrm{SRB}}\right) \leq \mathcal{P}_{\mathcal{G}_{H} \cap \mathcal{G}_{\mathcal{S}}},
$$

since we have identified a measure, namely $\hat{v}$, in $\mathcal{G}_{H} \cap \mathcal{G}_{\mathcal{S}}$ with $P_{\hat{v}}=\rho\left(\mu_{\mathrm{SRB}}\right)$. We will call upon the results in $\S 2.1$ to provide the reverse inequality-once we put ourselves in a viable setup. Notice that $\hat{v}=\pi_{*} \tilde{v}$ necessarily gives positive measure to $\Lambda=\pi\left(\Delta_{0}\right)$.

(a) $\quad \mu_{\mathrm{SRB}}=\varphi \mu$ with $\varphi \geq \delta>0$ on a neighborhood of $\Lambda$. In this case, $\hat{v} \in \mathcal{G}_{\varphi}$ since we can simply take the set $Z$ in the definition of $\mathcal{G}_{\varphi}$ to be this neighborhood. By

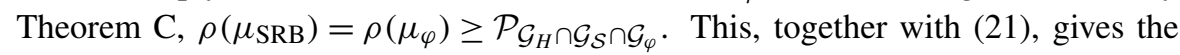
desired result.

(b) $\Lambda$ is contained in a $\mu_{\mathrm{SRB}}$-hyperbolic product set. Taking this set to be $\Pi$ in the definition of $\mathcal{G}_{\mathrm{SRB}}$, it is immediate that $\hat{v} \in \mathcal{G}_{\mathrm{SRB}}$. Theorem $\mathrm{C}$ and (21) then give the two halves of the desired equality.

Acknowledgement. The first author's research is partially supported by NSF grant DMS0801139.

\section{A. Appendix. Lyapunov charts for maps with singularities}

In this section, we prove the statements (a)(iii'), (b)(ii'), and (b)(iii') made in $\$ 4.2 .4$ regarding the Lyapunov charts $\left\{\Phi_{x}\right\}$. All notation is as in $\S 4$.

We begin with $v \in \mathcal{G}_{\mathcal{S}}$ and the set $V^{\prime}$ of regular points in the sense of Oseledec. Each $x \in V^{\prime}$ has $p$ distinct Lyapunov exponents $\lambda_{1}, \ldots, \lambda_{p}$ with corresponding subspaces $E_{1}(x), \ldots, E_{p}(x)$ such that $T_{x} M=\bigoplus_{i} E_{i}(x)$. Let $g_{\varepsilon}(x)=\frac{1}{3} \min \{\varepsilon, d(x, \mathcal{S})\}$.

Fix $\delta>0$. It follows by standard arguments (see [Y2, §3.1]) that for $v$-typical $x$, one can define an inner product, $\langle\cdot, \cdot\rangle_{x}^{\prime}$, on the tangent space $T_{x} M$ such that item (b)(i) of Proposition 4.1 holds. Denote by $\|\cdot\|_{x}^{\prime}$ the norm induced by $\langle\cdot, \cdot\rangle_{x}^{\prime}$, and by $\|\cdot\|_{x}$ the Euclidean norm on $T_{x} M$. It follows from the same construction that there exists a measurable function $\ell_{0}(x): V^{\prime} \rightarrow[1, \infty)$, with $\ell_{0}\left(f^{i} x\right)<e^{2 \delta i} \ell_{0}(x)$ for $i \geq 0$ and

$$
p^{-1 / 2}\|v\|_{x} \leq\|v\|_{x}^{\prime} \leq \ell_{0}(x)\|v\|_{x} \quad \text { for all } v \in T_{x} M \text {. }
$$

Define a linear map $L_{x}: T_{x} M \rightarrow \mathbb{R}^{d}$ which takes $E_{i}(x)$ to $\{0\} \times \cdots \times \mathbb{R}^{m_{i}(x)} \times \cdots \times\{0\}$ for each $i$ and such that $\left\langle L_{x} u, L_{x} v\right\rangle_{x}=\langle u, v\rangle_{x}^{\prime}$. Then $\Phi_{x}:=\exp _{x} \circ L_{x}^{-1}$ is a Lyapunov chart satisfying properties (a)(i) and (a)(ii) of Proposition 4.1.

The construction outlined thus far is standard and is not affected by the presence of singularities (see [KS, Part I, Theorem 2.2]). We now proceed to prove the statements of $\S 4.2 .4$ which are affected by singularities. We drop the subscript $x$ for simplicity of notation and write $\|\cdot\|$ and $\|\cdot\|^{\prime}$ in what follows.

Notice that in the notation of $\S 4, R(r)=R\left(r ;\|\cdot\|^{\prime}\right)$ denotes the ball of radius $r$ in the (Lyapunov) norm $\|\cdot\|^{\prime}$ since that is the norm of the Lyapunov charts $\Phi_{x}$. To distinguish between norms, we use $R(r ;\|\cdot\|)$ to denote the ball of radius $r$ in the Euclidean norm on $T_{x} M$. We identify $T_{x} M$ and $\mathbb{R}^{d}$ and view $L_{x}$ formally as a change of norm. 
Proof of $(a)\left(i i i^{\prime}\right)$. Recall the injectivity radius from $\S 2.1, \iota(x, U) \geq \min \left\{s, d(x, M \backslash U)^{\varsigma}\right\}$, given by equation (2). Since we have assumed $b \geq \varsigma$, we have $\iota(x, U) \geq g_{\varepsilon}(x)^{b}$ for $\varepsilon \leq s$. Thus, again using (2), for $y \in B\left(x, 0, g_{\varepsilon}(x)^{b}\right)$ and $w=\exp _{x}^{-1} y$, we have

$$
\left\|D\left(\exp _{x}\right)(w)\right\| \leq c_{0} \quad \text { and } \quad\left\|D\left(\exp _{x}^{-1}\right)(w)\right\| \leq c_{0} .
$$

This implies that $\exp _{x}$ maps $R\left(c_{0}^{-1} g_{\varepsilon}(x)^{b} ;\|\cdot\|\right)$ injectively into $B\left(x, 0, g_{\varepsilon}(x)^{b}\right)$. Thus, for $u, v \in R\left(c_{0}^{-1} g_{\varepsilon}(x)^{b} ;\|\cdot\|\right)$, we use (A1) to estimate

$$
\begin{aligned}
d\left(\Phi_{x} u, \Phi_{x} v\right) & \leq d\left(\exp _{x} \circ L_{x}^{-1} u, \exp _{x} \circ L_{x}^{-1} v\right) \leq c_{0}\left\|L_{x}^{-1} u-L_{x}^{-1} v\right\| \leq c_{0} \sqrt{p}\|u-v\|^{\prime} \\
& \leq c_{0} \sqrt{p} \ell_{0}(x)\|u-v\| \leq c_{0}^{2} \sqrt{p} \ell_{0}(x) d\left(\Phi_{x} u, \Phi_{x} v\right),
\end{aligned}
$$

which establishes (a)(iii') with $K=c_{0} \sqrt{p}$ and $\ell_{1}(x)=c_{0}^{2} \sqrt{p} \ell_{0}(x)$. Note that by (A1), $R\left(\ell_{1}^{-1}(x) g_{\varepsilon}(x)^{b}\right) \subseteq R\left(c_{0}^{-1} g_{\varepsilon}(x)^{b} ;\|\cdot\|\right)$, with room to spare.

Proof of $(b)\left(i i i^{\prime}\right)$. Recall that

$$
\hat{f}_{x}=\exp _{f x}^{-1} \circ f \circ \exp _{x} \quad \text { while } \tilde{f}_{x}=\Phi_{x}^{-1} \circ f \circ \Phi_{x}=L_{f x} \circ \hat{f}_{x} \circ L_{x}^{-1} .
$$

Taking $u, v, h \in R\left(c_{0}^{-1} g_{\varepsilon}(x)^{b} ;\|\cdot\|\right)$, we use (A1) to estimate

$$
\begin{aligned}
\frac{\left\|D \tilde{f}_{x}(u) h-D \tilde{f}_{x}(v) h\right\|^{\prime}}{\|h\|^{\prime}} & \leq \frac{\left\|D \hat{f}_{x}(u) h-D \hat{f}_{x}(v) h\right\|}{\|h\|} \sqrt{p} \ell_{0}(x) \\
& \leq\left\|D^{2} \hat{f}_{x}(z)\right\|\|u-v\| \sqrt{p} \ell_{0}(x)
\end{aligned}
$$

for some $z \in R\left(c_{0}^{-1} g_{\varepsilon}(x)^{b} ;\|\cdot\|\right)$. By (4), $\left\|D^{2} \hat{f}_{x}(z)\right\| \leq C_{1} d\left(\exp _{x}(z), \mathcal{S}\right)^{-b}$. Since $\exp _{x}(z) \in B\left(x, 0, g_{\varepsilon}(x)^{b}\right)$, we have $d\left(\exp _{x}(z), \mathcal{S}\right) \geq g_{\varepsilon}(x)$, so that $\left\|D^{2} \hat{f}_{x}(z)\right\| \leq$ $C_{1} g_{\varepsilon}(x)^{-b}$. Finally, since $\|u-v\| \leq \sqrt{p}\|u-v\|^{\prime}$, we conclude that

$$
\operatorname{Lip}\left(D \tilde{f}_{x}\right) \leq p \ell_{0}(x) C_{1} g_{\varepsilon}(x)^{-b} .
$$

The statement follows by taking $\ell(x)$ to be the larger of $p C_{1} \ell_{0}(x)$ and $\ell_{1}(x)=$ $c_{0}^{2} \sqrt{p} \ell_{0}(x)$.

Proof of $(b)\left(i i^{\prime}\right)$. We use (A2) with $v=0$ and $u \in R\left(\delta \ell(x)^{-1} g_{\varepsilon}(x)^{b}\right)$. This yields

$$
\left\|D \tilde{f}_{x}(u)-D \tilde{f}_{x}(0)\right\|^{\prime} \leq \ell(x) g_{\varepsilon}(x)^{-b}\|u\|^{\prime} \leq \delta .
$$

This implies that, restricted to $R\left(\delta \ell(x)^{-1} g_{\varepsilon}(x)^{b}\right)$, we have $\operatorname{Lip}\left(\tilde{f}_{x}-D \tilde{f}_{x}(0)\right) \leq \delta$ as required.

\section{B. Appendix. Natural extensions of tower maps}

Let $T:(X, \Sigma, v) \circlearrowleft$ be a measure-preserving transformation (mpt) of a probability space. Recall that the natural extension of $T:(X, \Sigma, v) \circlearrowleft$, denoted here by $T^{\sharp}:\left(X^{\sharp}, \Sigma^{\sharp}, v^{\sharp}\right) \circlearrowleft$, is defined as follows:

$$
\begin{gathered}
X^{\sharp}=\left\{\left(x_{1}, x_{2}, \ldots\right) \in \prod_{i=0}^{\infty} X: T\left(x_{i+1}\right)=x_{i}\right\}, \\
T^{\sharp}\left(x_{1}, x_{2}, \ldots\right)=\left(T\left(x_{1}\right), x_{1}, x_{2}, \ldots\right) .
\end{gathered}
$$


$\Sigma^{\sharp}$ is generated by cylinder sets with $\Sigma$ in each coordinate, and

$$
\nu^{\sharp}\left\{x_{1} \in A_{1}, \ldots, x_{n} \in A_{n}\right\}=v\left(A_{n} \cap T^{-1} A_{n-1} \cap \cdots \cap T^{-(n-1)} A_{1}\right) .
$$

These following facts about tower maps (see $\$ 5.1$ for notation) are used.

(1) Consider $F:\left(\Delta^{\infty}, \Sigma, \eta\right) \circlearrowleft$, where $\eta$ is any $F$-invariant Borel probability measure, and let $\bar{F}:\left(\bar{\Delta}^{\infty}, \bar{\Sigma}, \bar{\eta}\right) \circlearrowleft$ be the corresponding quotient system. We claim that the natural extensions of these two mpt's are isomorphic.

Proof. Define $\bar{\pi}^{\sharp}: \Delta^{\sharp} \rightarrow \bar{\Delta}^{\sharp}$ by $\bar{\pi}^{\sharp}\left(x_{1}, x_{2}, \ldots\right)=\left(\bar{\pi}\left(x_{1}\right), \bar{\pi}\left(x_{2}\right), \ldots\right)$. Clearly, $\bar{\pi}^{\sharp} 。$ $F^{\sharp}=\bar{F}^{\sharp} \circ \bar{\pi}^{\sharp}, \bar{\pi}_{*}^{\sharp}\left(\eta^{\sharp}\right)=\bar{\eta}^{\sharp}$, and $\bar{\pi}^{\sharp}$ is onto. The assertion follows once we show $\bar{\pi}^{\sharp}$ is one-to-one.

Suppose $\bar{\pi}^{\sharp}\left(x_{1}, x_{2}, \ldots\right)=\bar{\pi}^{\sharp}\left(y_{1}, y_{2}, \ldots\right)$. Letting $\omega^{s}\left(x_{n}\right)$ denote the stable set of $x_{n}$, we have, by definition, $x_{1} \in \bigcap_{n=1}^{\infty} F^{n-1}\left(\omega^{s}\left(x_{n}\right)\right)$. The uniform contraction of $F$ along stable sets implies that this intersection consists of a single point. Likewise, $\left\{y_{1}\right\}=\bigcap_{n=1}^{\infty} F^{n-1}\left(\omega^{s}\left(y_{n}\right)\right)$. Since $\bar{\pi}\left(x_{n}\right)=\bar{\pi}\left(y_{n}\right)$ is equivalent to $\omega^{s}\left(x_{n}\right)=\omega^{s}\left(y_{n}\right)$, we have proved $x_{1}=y_{1}$. Applying the same argument to the sequences $\left(x_{k}, x_{k+1}, \ldots\right)$ and $\left(y_{k}, y_{k+1}, \ldots\right)$, we conclude that $x_{k}=y_{k}$ for all $k \geq 1$.

(2) Next, given $\bar{F}:\left(\bar{\Delta}^{\infty}, \bar{\Sigma}, \bar{\eta}\right) \circlearrowleft$ and $\bar{\Delta}_{0}^{\infty} \subset \bar{\Delta}^{\infty}$, we call $\bar{F}^{R}:\left(\bar{\Delta}_{0}^{\infty}, \bar{\Sigma}_{0}, \bar{\eta}_{0}\right) \circlearrowleft$ with $\bar{\eta}_{0}=\left.\bar{\eta}\right|_{\bar{\Delta}_{0}}$ normalized its induced map on $\bar{\Delta}_{0}$, and claim that the induced map of $\bar{F}^{\sharp}$ on $\bar{\Delta}_{0}^{\sharp}=\left\{\left(x_{1}, x_{2}, \ldots\right) \in \bar{\Delta}^{\sharp}: x_{1} \in \bar{\Delta}_{0}\right\}$ is the natural extension of $\bar{F}^{R}$. The proof is easy.

FACT. For an arbitrary mpt $T:(X, \mathcal{A}, v) \circlearrowleft$, it is proved in $[\mathbf{R o}]$ that $h_{v}(T)=h_{v^{\sharp}}\left(T^{\sharp}\right)$.

\section{REFERENCES}

[A] L. M. Abramov. The entropy of a derived automorphism. Dokl. Akad. Nauk. SSSR 128 (1959), 647-650; Amer. Math. Soc. Transl. 49(2) (1966), 162-166.

[B] R. Bowen. Equilibrium States and the Ergodic Theory of Anosov Diffeomorphisms (Lecture Notes in Mathematics, 470). Springer, Berlin, 1975.

[BK] M. Brin and A. Katok. On local entropy. Geometric Dynamics (Rio de Janeiro, 1981) (Lecture Notes in Mathematics, 1007). Springer, Berlin, 1983, pp. 30-38.

[BDM] H. Bruin, M. Demers and I. Melbourne. Existence and convergence properties of physical measures for certain dynamical systems with holes. Ergod. Th. \& Dynam. Sys. 30 (2010), 687-728.

[Bu] J. Buzzi. Markov extensions for multidimensional dynamical systems. Israel J. Math. 112 (1999), 357-380.

[CM1] N. Chernov and R. Markarian. Ergodic properties of Anosov maps with rectangular holes. Bol. Soc. Bras. Mat. 28 (1997), 271-314.

[CM2] N. Chernov and R. Markarian. Anosov maps with rectangular holes. Non-ergodic cases. Bol. Soc. Bras. Mat. 28 (1997), 315-342.

[CM3] N. Chernov and R. Markarian. Chaotic Billiards (Mathematical Surveys and Monographs, 127). American Mathematical Society, Providence, RI, 2006.

[CMT] N. Chernov, R. Markarian and S. Troubetzkoy. Invariant measures for Anosov maps with small holes. Ergod. Th. \& Dynam. Sys. 18 (1998), 1049-1073.

[CMS] P. Collet, S. Martínez and B. Schmitt. The Yorke-Pianigiani measure and the asymptotic law on the limit Cantor set of expanding systems. Nonlinearity 7 (1994), 1437-1443.

[D] M. F. Demers. Functional norms for Young towers. Ergod. Th. \& Dynam. Sys. 30(5) (2010), 1371-1398. 
[DWY] M. F. Demers, P. Wright and L.-S. Young. Escape rates and physically relevant measures for billiards with small holes. Comm. Math. Phys. 294 (2010), 353-388.

[DY] M. F. Demers and L.-S. Young. Escape rates and conditionally invariant measures. Nonlinearity 19 (2006), 377-397.

[KS] A. Katok and J.-M. Strelcyn. Invariant Manifolds, Entropy and Billiards; Smooth Maps with Singularities (Lecture Notes in Mathematics, 1222). Springer, Berlin, 1986, p. 283; with the collaboration of F. Ledrappier and F. Przytycki.

[LY] F. Ledrappier and L.-S. Young. The metric entropy of diffeomorphisms. Part I: characterization of measures satisfying Pesin's entropy formula. Ann. of Math. (2) 122 (1985), 509-539.

[M] R. Mañé. A proof of Pesin's formula. Ergod. Th. \& Dynam. Sys. 1(1) (1981), 95-102.

[P] Ya. B. Pesin. Characteristic Lyapunov exponents and smooth ergodic theory. Russian Math. Surveys 32(4) (1977), 55-114.

[Pe] K. E. Petersen. Ergodic Theory (Cambridge Studies in Advanced Mathematics). Cambridge University Press, Cambridge, 1989, p. 329.

[Ro] V. Rohklin. Exact endomorphisms of Lebesgue space. Amer. Math. Soc. Transl. 239 (1964), 1-36.

[R] D. Ruelle. An inequality for the entropy of differentiable maps. Bol. Soc. Bras. Mat. 9(1) (1978), 83-87.

[S] O. Sarig. Thermodynamic formalism of countable Markov shifts. Ergod. Th. \& Dynam. Sys. 19 (1999), 1565-1593.

[W] P. Walters. An Introduction to Ergodic Theory (Graduate Texts in Mathematics, 79). Springer, New York, 1982, p. 250.

[Y1] L.-S. Young. Some large deviation results for dynamical systems. Trans. Amer. Math. Soc. 318(2) (1990), 525-543.

[Y2] L.-S. Young. Ergodic theory of differentiable dynamical systems. Real and Complex Dynamics (NATO ASI Series, C464). Eds. B. Branner and P. Hjorth. Kluwer Academic Publishers, Dordrecht, 1995, pp. 293-336.

[Y3] L.-S. Young. Statistical properties of dynamical systems with some hyperbolicity. Ann. of Math. (2) 147(3) (1998), 585-650.

[Y4] L.-S. Young. What are SRB measures and which dynamical systems have them? J. Stat. Phys. 108 (2002), 733-754. 